Publ. RIMS, Kyoto Univ.

21 (1985), 47-74

\title{
Stability Changes of Periodic Solutions \\ to a Coupled Nonlinear Equation \\ with Time Delay
}

By

Yoshihisa MORITA*

\begin{abstract}
We shall consider a linear difference coupled equation with time delay and discuss a Hopf bifurcation of this equation. In the case of weakly coupling, it will be shown that two types of periodic solutions bifurcate from the steady state for some parameter values, and that those periodic solutions exchange the stability under certain conditions; moreover, under another conditions one of those periodic solutions changes its stability twice at least. Sufficient conditions for the occurrence of such phenomena will be presented along with specific examples.
\end{abstract}

\section{Introduction}

Many variety of oscillatory phenomena appear in electronics, biology and chemistry. Models which describe such phenomena have been proposed by using ordinary and partial differential equations, also differential equations with time delay. Hutchinson [9] has introduced the following differential equation with time delay as a biological model, representing oscillatory phenomenon occurring in the growth process of a single species:

$$
\frac{d}{d s} y(s)=a\left(1-\frac{y(s-r)}{K}\right) y(s), \quad a, K, r>0
$$

Put $x(t)=\frac{y(r t)}{K}-1$. Then this equation can be transformed into

$$
\frac{d}{d t} x(t)=-\alpha(1+x(t)) x(t-1)
$$

* Communicated by S. Matsuura, December 9, 1983. Revised April 18, 1984.

Research Institute for Mathematical Sciences, Kyoto University, Kyoto 606, Japan. 
where $\alpha=a r$, and the steady state $y \equiv K$ of $(\mathbb{E} 1)$ corresponds to $x \equiv 0$ of $(\mathbb{E} 2)$. It is known that for $\alpha>\pi / 2$ the equation (E2) has a periodic solution, as first proved by Jones [11]. Also one will find in [3] and [8] that when the parameter $\alpha$ passes through the value $\pi / 2$ a Hopf bifurcation occurs and that the bifurcating periodic solution from the steady state $x \equiv 0$ is stable near the bifurcation point.

In this paper, using the above equation (E2), we shall consider the following linear difference coupled equation:

$$
\left\{\begin{array}{l}
\frac{d}{d t} x^{1}(t)=\nu\left(x^{2}(t)-x^{1}(t)\right)-\left(\frac{\pi}{2}+\mu\right)\left(1+x^{1}(t)\right) x^{1}(t-1) \\
\frac{d}{d t} x^{2}(t)=\nu\left(x^{1}(t)-x^{2}(t)\right)-\left(\frac{\pi}{2}+\mu\right)\left(1+x^{2}(t)\right) x^{2}(t-1)
\end{array}\right.
$$

where $\alpha=\frac{\pi}{2}+\mu, \nu \geqq 0$; we shall focus on a bifurcation problem for a periodic solution to $(\mathbb{E} 3)$, and discuss the stability change of the bifurcating periodic solution.

It is clear that for $\mu>0(\mathbb{E} 3)$ has a periodic solution $\left(x^{1}(t), x^{2}(t)\right)=(\tilde{p}(t)$, $\tilde{p}(t))$, which is called the in-phase solution, where $\tilde{p}(t)$ is a periodic solution to $(\mathbb{E} 2)$. We also see that another periodic solution to $(\mathbb{E} 3)$ bifurcates from the steady state $\left(x^{1}, x^{2}\right)=(0,0)$ for suitable parameter values $\mu$ and $\nu$. This periodic solution is called the anti-phase solution to $(\mathbb{E} 3)$ (for the details, see $\S 3$ ).

By the usual Hopf bifurcation theorem (see [3] and [8]), we can discuss the stability of those bifurcating periodic solutions. Actually, for any fixed positive $\nu$, letting $\mu$ be a bifurcation parameter, we see the stability of bifurcating solutions to (E3) near the bifurcation points. For example, for each positive $\nu$ the in-phase solution of $(\mathbb{E} 3)$ is virtually stable on some interval of $\mu$. However, the stability region of $\mu$ (that is, the set of all $\mu$ for which the bifurcating solution is stable) may vary according to $\nu$, even if it continues to be non-empty. This suggests the possibility of the occurrence of stability change, which might be observed when we vary $\nu$.

In this paper we shall discuss this. In the equation (E3), applying Theorem 1 obtained in $\S 2$ yields that the stability region of the in-phase solution shrinks as $\nu$ tends to zero ; that is, for sufficiently small $\nu$, the in-phase solution to (E3) loses its stability fairly near the bifurcation point. Moreover, then we see from Corollary 7 in $\S 4$ that the anti-phase solution to $(\mathbb{E} 3)$ recovers its stability for suitable parameter values $\mu$ and $\nu$; hence those solutions exchange the stability.

We will formulate the differential delay equations $(\mathbb{E} 2)$ and $(\mathbb{E} 3)$ in general form 
of functional differential equation (see (1.1) and (1.2)), and discuss the bifurcation of the in-phase and the anti-phase solutions of (1.1). Next we shall study the linearized stability of those periodic solutions in quite a general framework. Corollary 7 in $\$ 4$ gives sufficient conditions for the occurrence of exchange of the stability, for $(\mu, \nu)$ sufficiently small. And from Corollary 8 in $\S 4$ one will see that the anti-phase solution changes its stability twice under another conditions.

The studies of two coupled systems of nonlinear oscillators using ordinary differential equations (without time delay) will be found in plenty of works including [1], [5], [14], [15], [21] [23]. The occurrence of stability change of the in-phase or anti-phase solution to such coupled systems has been already suggested by perturbation methods and numerical experiments. One will also see that the same arguments in the case of functional differential equations can apply to that of linear difference coupled systems using ordinary differential equations (without time delay), provided that one replaces function spaces, inner products and other notations by appropriate ones. This application with a specific example (biochemical model, the Brusselator) will be found in $\S 5$; Example 2.

The author would like to express his gratitude to Professor Masaya Yamaguti for his continued encouragement. He also expresses his sincere acknowledgement to Professor Masayasu Mimura for his stimulating discussions.

\section{§1. A Limear $\mathbb{D}$ inferemee Coupled Equation wirk

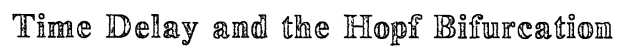

Let $\mathbb{R}^{m}$ be the $m$-dimensional Euclid space, and let $\mathbb{C}\left([a, b] ; \mathbb{R}^{m}\right)$ be the set of all continuous functions defined on $[a, b]$ with values in $\mathbb{R}^{m}$.

$\mathbb{C}\left([a, b] ; \mathbb{R}^{m}\right)$ is a Banach space equipped with supremum norm $\|\bullet\|$. We simply write $\mathbb{C}[a, b]$ instead of $\mathbb{C}\left([a, b] ; \mathbb{R}^{m}\right)$. For any $x \in \mathbb{C}[a-r, b]$ and $t \in[a, b]$ the notation $x_{t}$ will denote the element in $\mathbb{C}[-r, 0]$ defined by $x_{t}(\theta) \equiv x(t+\theta),-r \leqq$ $\theta \leqq 0$, where $r$ is a positive number.

Let a function $F\left({ }^{\circ},{ }^{\circ}\right)$ be a sufficiently smooth function of $\mathbb{R} \times \mathbb{C}[-r, 0]$ into $\mathbb{R}^{m}$, which satisfies

$$
\mathbb{F}(\mu, 0)=0 \quad \text { for } \mu \in \mathbb{I}_{0}
$$

where $\mathbb{I}_{0}$ is an open interval containing the origin. The notation $D$ will denote a continuous linear operator of $C[-r, 0]$ into $\mathbb{R}^{m}$. 
Now we shall consider the following functional differential equation:

$$
\left(\begin{array}{c}
\dot{x}^{1}(t) \\
\dot{x}^{2}(t)
\end{array}\right)=\left(\begin{array}{l}
F\left(\mu, x_{t}^{1}\right)+\nu D\left[x_{t}^{2}-x_{t}^{1}\right] \\
F\left(\mu, x_{t}^{2}\right)+\nu D\left[x_{t}^{1}-x_{t}^{2}\right]
\end{array}\right), \quad \nu \geqq 0,
$$

where $\cdot$ denotes $\frac{d}{d t}$. We call the equation (1.1) a linear difference coupled equation of

$$
\dot{x}(t)=F\left(\mu, x_{t}\right)=L(\mu) x_{t}+G\left(\mu, x_{t}\right),
$$

where $L(\mu)$ and $G\left(\mu,{ }^{\circ}\right)$ are the linear part and the higher order nonlinear part of $F(\mu, \circ)$ respectively.

In this section we shall discuss a bifurcation of a periodic solution to (1.1) from the trivial solution under suitable conditions. Theorems for a $\mathbb{H}$ opf bifurcation found in [3], [7] and [8] might apply to (1.1). Their methods, however, can not make clear whether or not the bifurcating periodic solution changes its stability. We also discuss the occurrence of the stability change in the later sections.

First let us consider the linearized equation of (1.1) around the steady state $\left(x^{1}, x^{2}\right)=(0,0)$,

$$
\left(\begin{array}{c}
\dot{y}^{1}(t) \\
\dot{y}^{2}(t)
\end{array}\right)=\left(\begin{array}{l}
L(\mu) y_{t}^{1}+\nu D\left[y_{t}^{2}-y_{t}^{1}\right] \\
L(\mu) y_{t}^{2}+\nu D\left[y_{t}^{1}-y_{t}^{2}\right]
\end{array}\right), \nu \geqq 0,
$$

which is also written as

$$
\left(\begin{array}{l}
\dot{y}^{1}(t) \\
\dot{y}^{2}(t)
\end{array}\right)=\left(\begin{array}{cc}
L(\mu) & 0 \\
0 & L(\mu)
\end{array}\right)\left(\begin{array}{l}
y_{t}^{1}(t) \\
y_{t}^{2}(t)
\end{array}\right)+\left(\begin{array}{rr}
-\nu D & \nu D \\
\nu D & -\nu D
\end{array}\right)\left(\begin{array}{l}
y_{t}^{1}(t) \\
y_{t}^{2}(t)
\end{array}\right)
$$

After the change of variables

$$
\left(\begin{array}{c}
w_{t}^{1} \\
w_{t}^{2}
\end{array}\right)=\left(\begin{array}{rr}
\frac{1}{2} & \frac{1}{2} \\
\frac{1}{2} & -\frac{1}{2}
\end{array}\right)\left(\begin{array}{c}
y_{t}^{1} \\
y_{t}^{2}
\end{array}\right),
$$

(1.4) is transformed into

$$
\left(\begin{array}{l}
\dot{w}^{1}(t) \\
\dot{w}^{2}(t)
\end{array}\right)=\left(\begin{array}{c}
\mathbb{L}(\mu) w_{t}^{1} \\
(\mathbb{L}(\mu)-2 \nu D) w_{t}^{2}
\end{array}\right)
$$

As $L(\mu)$ and $D$ are continuous linear operators of $\mathbb{C}[-r, 0]$ into $\mathbb{R}^{m}$, by $\mathbb{R}$ iesz' 
representation theorem there are $m \times m$ matrices functions $\eta(\theta ; \mu)$ and $\eta^{\prime}(\theta),-r \leqq$ $\theta \leqq 0$, whose elements have bounded variation in $\theta$ on $[-r, 0]$, such that

$$
\begin{gathered}
\mathbb{L}(\mu) \phi=\int_{-r}^{0}[d \eta(\theta ; \mu)] \phi(\theta), \quad \phi \in \mathbb{C}[-r, 0], \\
D[\phi]=\int_{-r}^{0}\left[d \eta^{\prime}(\theta)\right] \phi(\theta), \quad \phi \in \mathbb{C}[-r, 0] .
\end{gathered}
$$

Let $\mathbb{C}^{m}$ be the $m$-dimensional complex space. The domain $\mathbb{C}[-r, 0]$ of $L(\mu)$ and $D$ is naturally extended into $C\left([-r, 0] ; \mathbb{C}^{m}\right)$, and $(1.7 \mathrm{a}),(1.7 \mathrm{~b})$ hold for $\phi \in$ $\mathbb{C}\left([-r, 0] ; \mathbb{C}^{m}\right)$. Hereafter we also denote $\mathbb{C}\left([-r, 0] ; \mathbb{C}^{m}\right)$ by $\mathbb{C}[-r, 0]$ as long as there is no confusion.

The characteristic equations of (1.6) are defined by

$$
\begin{gathered}
\operatorname{det}\left(\lambda I-\int_{-r}^{0}[d \eta(\theta ; \mu)] e^{\lambda \theta}\right)=0, \\
\operatorname{det}\left(\lambda I-\int_{-r}^{0}[d \tilde{\eta}(\theta ; \mu, \nu)] e^{\lambda \theta}\right)=0, \\
\tilde{\eta}(\theta ; \mu, \nu) \risingdotseq \eta(\theta ; \mu)-2 \nu \eta^{\prime}(\theta), \quad-r \leqq \theta \leqq 0,
\end{gathered}
$$

where I denotes the $m \times m$ identity matrix. The root of (1.8) gives the eigenvalue of the equation (1.6) (or (1.3)) (see Hale [7; Chap. 7]).

We assume that

(A1) (1.8a) has a pair of simple complex conjugate roots $\lambda(\mu), \overline{\lambda(\mu)}$ such that

$$
\lambda(0)=i \omega_{0}\left(\omega_{0}>0\right), \operatorname{Re} \frac{d \lambda}{d \mu}(0) \neq 0 ;
$$

(A2) the remaining roots of (1.8a) have strictly negative real paris.

Since (1.8a) is the characteristic equation for

$$
\dot{w}(t)=\mathbb{L}(\mu) w_{t}, \quad t>0,
$$

which is the linearized equation of (1.2), from the above assumptions (A1) and (A2) it follows that in the equation (1.2) a Hopf bifurcation occurs at $\mu=0$; that is, there is a family of periodic solutions which bifurcate from the zero solution in a neighborhood of $\mu=0$. (See Hale [7; Chap. 11].) Then we also see that a periodic solution to (1.1) bifurcates from the steady state $\left(x^{1}, x^{2}\right)=(0,0)$ at $\mu=0$ because $(p(t), p(t))$ is a solution to (1.1) for a solution $p(t)$ to (1.2). 
Next consider the equation (1.8b). From Appendix $\mathrm{A}$ we see that there is a number $\nu_{r}$, and there exist functions,

$$
\mu=\mu_{0}(\nu)\left(\mu_{0}(0)=0\right), \quad \alpha=\alpha_{0}(\nu)\left(\alpha_{0}(0)=\omega_{0}\right), \quad \nu \in\left[0, \nu_{r}\right]
$$

such that for $\nu \in\left[0, \nu_{r}\right]$ and $\mu=\mu_{0}(\nu)$ the equation (1.8b) has the simple roots, $\pm i \alpha_{0}(\nu)$. Let $\tilde{\lambda}(\mu, \nu)$ be the roots of $(1.8 \mathrm{~b})$ which satisfies $\tilde{\lambda}\left(\mu_{0}(\nu), \nu\right)=i \alpha_{0}(\nu)$. By the assumption (Al) there exists a number $\bar{\nu}, 0<\bar{\nu}<\nu_{r}$, such that

$$
\frac{\partial \tilde{\lambda}}{\partial \mu}\left(\mu_{0}(\nu), \nu\right) \neq 0 \quad \text { for } \nu \in[0, \bar{\nu}] \equiv \mathbb{I}_{\nu}
$$

If $\alpha_{0}(\nu) \neq \omega_{0}(\nu \in(0, \bar{\nu}])$, by letting $\mu$ be a bifurcation parameter, the Hopf bifurcation theorem in [7] can apply to the equation (1.1); there exists a periodic solution bifurcating from the zero solution at $\mu=\mu_{0}(\nu)$.

Thus we can obtain two types of periodic solutions to (1.1) bifurcating from the zero solution. In the rest of this paper we shall discuss the stability.

To simplify our notation for the derivatives of $F(\mu, u)$, in this paper we write

$$
\begin{aligned}
& F_{u}(\mu) \equiv \frac{\partial}{\partial u} F(\mu, 0) \quad(=L(\mu)), \\
& F_{\mu u}(\cdot) \equiv \frac{\partial^{2}}{\partial \mu \partial u} F(\mu, 0) \quad\left(=\frac{\partial}{\partial \mu} L(\mu)\right), \\
& F_{u u}(\mu)(\cdot, \cdot) \equiv \frac{\partial^{2}}{\partial u^{2}} F(\mu, 0)(\cdot, \cdot), \\
& F_{u u u}(\mu)(\cdot, \cdot, \cdot) \equiv \frac{\partial^{3}}{\partial u^{3}} F(\mu, 0)(\cdot, \cdot, \cdot),
\end{aligned}
$$

and so forth. Furthermore, before concluding this section, we introduce some notation and function spaces which will be used in later arguments.

Let $\xi_{0}(\nu)$ and $\xi_{0}^{*}(\nu)$ be vectors satisfying

$$
\begin{gathered}
\left(i \alpha_{0}(\nu) I-\int_{-r}^{0} e^{i \alpha_{0}(\nu) \theta}\left[d \tilde{\eta}\left(\theta ; \mu_{0}(\nu), \nu\right)\right]\right) \xi_{0}(\nu)=0, \\
\left(-i \alpha_{0}(\nu) I-\int_{-r}^{0} e^{-i \alpha_{0}(\nu) \theta t}\left[d \tilde{\eta}\left(\theta ; \mu_{0}(\nu), \nu\right)\right]\right) \xi_{0}^{*}(\nu)=0,
\end{gathered}
$$

respectively, where ${ }^{t}[d \tilde{\eta}(\cdot ; \cdot, \cdot)]$ denotes the transpose of $\left[d \tilde{\eta}\left(\bullet^{\circ} ;{ }^{\circ}, \cdot\right)\right]$.

Define the (formal) product as 


$$
\begin{aligned}
\langle\phi, \phi\rangle_{\nu} \equiv(\phi(0), \phi(0))-\int_{-r}^{0} \int_{0}^{\theta t} \overline{\phi(\xi-\theta)}\left[d \tilde{\eta}\left(\theta ; \mu_{0}(\nu), \nu\right)\right] \phi(\xi) d \xi \\
\quad \text { for } \phi \in \mathbb{C}[-r, 0] \text { and } \phi \in \mathbb{C}[0, r]
\end{aligned}
$$

where $\left(\cdot,{ }^{\circ}\right)$ is the hermite product in $\mathbb{C}^{m}$, i.e.,

$$
(a, b)={ }^{t} \bar{b} a=\sum_{i=1}^{n} a_{i} \bar{b}_{i}, a={ }^{t}\left(a_{1}, \cdots, a_{m}\right), b={ }^{t}\left(b_{1}, \cdots, b_{m}\right)
$$

We let

$$
\begin{aligned}
& {\left[\xi_{1}(\nu)\right](\theta) \equiv \xi_{0}(\nu) e^{i \alpha_{0}(\nu) \theta}, \quad-r \leqq \theta \leqq 0,} \\
& {\left[\xi_{1}^{*}(\nu)\right](\theta) \equiv \xi_{0}^{*}(\nu) e^{i \alpha_{0}(\nu) \theta}, \quad 0 \leqq \theta \leqq r .}
\end{aligned}
$$

Then we can take $\xi_{1}(\nu)$ and $\xi_{1}^{*}(\nu)$ as satisfy

$$
\left\langle\xi_{1}(\nu), \xi_{1}^{*}(\nu)\right\rangle_{\nu}=1, \quad \nu \in \mathbb{I}_{\nu}
$$

Furthermore,

$$
\begin{gathered}
\left\langle\xi_{1}(\nu), \overline{\xi_{1}^{*}(\nu)}\right\rangle_{\nu}=\left\langle\overline{\xi_{1}(\nu)}, \xi_{1}^{*}(\nu)\right\rangle_{\nu}=0, \\
\left\langle\xi_{1}(\nu), \xi_{1}^{*}(\nu)\right\rangle_{\nu}=\left(M(\nu) \xi_{0}(\nu), \xi_{0}^{*}(\nu)\right), \\
M(\nu) \equiv I-\int_{-r}^{0} \theta e^{i \alpha_{0}(\nu) \theta}\left[d \tilde{\eta}\left(\theta ; \mu_{0}(\nu), \nu\right)\right]
\end{gathered}
$$

hold (see [7; Chap. 7]).

$\xi_{2,0}(\nu)$ and $\widehat{\xi}_{2,0}(\nu)$ will denote the vectors determined uniquely by the equations,

$$
\begin{gathered}
\left(2 i \alpha_{0}(\nu)-L\left(\mu_{0}(\nu)\right)\left(e^{2 i \alpha_{0}(\nu) \cdot}\right)\right) \xi_{2,0}(\nu) \\
=\frac{1}{2} F_{u u}\left(\mu_{0}(\nu)\right)\left(\xi_{1}(\nu), \xi_{1}(\nu)\right), \\
-L\left(\mu_{0}(\nu)\right) \widehat{\xi}_{2,0}(\nu)=F_{u u}\left(\mu_{0}(\nu)\right)\left(\xi_{1}(\nu), \overline{\xi_{1}(\nu)}\right),
\end{gathered}
$$

respectively, and define

$$
\begin{aligned}
& {\left[\xi_{2}(\nu)\right](\theta) \equiv \xi_{2,0}(\nu) e^{2 i \alpha_{0}(\nu) \theta},} \\
& {\left[\widehat{\xi}_{2}(\nu)\right](\theta) \equiv \widehat{\xi}_{2,0}(\nu), \quad-r \leqq \theta \leqq 0}
\end{aligned}
$$

When $\nu=0$, we write 


$$
\zeta_{0} \equiv \xi_{0}(0), \quad \zeta_{0}^{*} \equiv \xi_{0}^{*}(0), \quad \zeta_{1} \equiv \xi_{1}(0), \quad \zeta_{1}^{*} \equiv \xi_{1}^{*}(0),
$$

$$
\zeta_{2,0} \risingdotseq \xi_{2,0}(0), \quad \widehat{\zeta}_{2,0} \risingdotseq \widehat{\xi}_{2,0}(0), \quad \zeta_{2} \equiv \xi_{2}(0), \quad \widehat{\zeta}_{2} \equiv \widehat{\xi}_{2}(0) \text {. }
$$

By the smoothness of $\xi_{0}(\nu), \xi_{0}^{*}(\nu), \xi_{2,0}(\nu)$ and $\widehat{\xi}_{2,0}(\nu)$ with respect to $\nu$, for sufficiently small $\nu$ we obtain

$$
\begin{cases}\xi_{0}(\nu)=\zeta_{0}+o(\nu), & \xi_{0}^{*}(\nu)=\zeta_{0}^{*}+o(\nu) \\ \xi_{2,0}(\nu)=\xi_{2,0}+o(\nu), & \bar{\xi}_{2,0}(\nu)=\hat{\zeta}_{2,0}+o(\nu)\end{cases}
$$

Finally we define the function spaces $P_{2 \pi}$ and $P_{2 \pi}^{(1)}$ as

$$
\begin{gathered}
\mathbb{P}_{2 \pi} \equiv\left\{u \mid u \in \mathrm{C}\left([0,2 \pi] ; \mathbb{C}^{m}\right), u(s+2 \pi)=u(s)\right\}, \\
\mathbb{P}_{2 \pi}^{(1)} \risingdotseq\left\{u \mid u, \frac{d u}{d s} \in \mathbb{P}_{2 \pi}\right\} .
\end{gathered}
$$

$P_{2 \pi}$ and $P_{2 \pi}^{(1)}$ are Banach spaces with norms $\|u\|=\sup _{0 \leq s \leq 2 \pi}|u(s)|, u \in P_{2 \pi},\|u\|=\max$ $\left\{\|u\|,\left\|\frac{d u}{d s}\right\|\right\}, u \in P_{2 \pi}^{(1)}$, respectively.

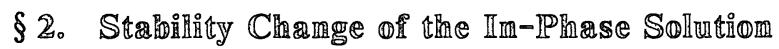

As seen in the preceding section, under the assumptions (A1) and (A2) (1.1) has a periodic solution which bifurcates from $(0,0)$ at $\mu=0$. This periodic solution has the form $\left(x^{1}(t), x^{2}(t)\right)=(p(t), p(t))$, where $p(t)$ is a periodic soution to (1.2). We call this periodic solution the in-phase solution to (1.1). It is clear that the in-phase solution to (1.1) exists for $\mu$ in some interval independent of the parameter $\nu$.

In the equation (1.2), applying the Hopf bifurcation theorem yields a family of periodic solution $p(t ; \varepsilon), 0<\varepsilon<\varepsilon_{H}$, which exists for $\mu=\mu(\varepsilon)$ and has period $T=$ $2 \pi / \omega(\varepsilon)$, where $\mu(\varepsilon)$ and $\omega(\varepsilon)$ are smooth functions in $\varepsilon$ having the forms

$$
\left\{\begin{array}{l}
\mu(\varepsilon)=\mu_{2} \varepsilon^{2}+O\left(\varepsilon^{3}\right) \\
\omega(\varepsilon)=\omega_{0}+\omega_{2} \varepsilon^{2}+O\left(\varepsilon^{3}\right)
\end{array}\right.
$$

Moreover, the above periodic solution has the Floquet exponents 0 and $\beta=\beta(\varepsilon)$, where $\beta(\varepsilon)$ is given by

$$
\left\{\begin{array}{l}
\beta(\varepsilon)=\beta_{2} \varepsilon^{2}+\mathcal{O}\left(\varepsilon^{3}\right), \\
\beta_{2}=-2 \mu_{2} \operatorname{Re} \frac{d \lambda}{d \mu}(0)
\end{array}\right.
$$


(See [8] and [19].) Therefore, if $\beta_{2}<0$, then there is a number $\varepsilon_{0}, 0<\varepsilon_{0}<\varepsilon_{H}$, such that for $\varepsilon \in\left(0, \varepsilon_{0}\right)$ the periodic solution $p(\circ ; \varepsilon)$ to $(1.2)$ is "asymptotically stable" (hereafter we simply say "stable"). In the present paper we assume

$$
\beta_{2}<0 \text {. }
$$

Under the assumption $(\mathbb{A} 3)$ the in-phase solution $\left(x^{1}(t), x^{2}(t)\right)=(p(t ; \varepsilon)$, $p(t ; \varepsilon))$ to $(1.1)$ is stable with respect to homogeneous perturbation, however, it is not clear whether or not the in-phase solution is stable with respect to inhomogeneous perturbation; where the homogeneous perturbation means perturbation having equal components. Therefore, to discuss the stability of the in-phase solution, we shall investigate Floquet exponents of the linearized equation of (1.1) around the solution.

After adopting new variables $s=\omega(\varepsilon) t, y^{i}(s)=x^{i}(t)(i=1,2),(1.1)$ is written as

$$
\begin{aligned}
\omega(\varepsilon) & \frac{d}{d s}\left(\begin{array}{l}
y^{1}(s) \\
y^{2}(s)
\end{array}\right) \\
= & \left(\begin{array}{l}
\mathbb{F}\left(\mu(\varepsilon), y_{s, \omega(\varepsilon)}^{1}\right)+\nu \mathbb{D}\left[y_{s, \omega(\varepsilon)}^{2}-y_{s, \omega(\varepsilon)}^{1}\right] \\
\mathbb{F}\left(\mu(\varepsilon), y_{s, \omega(\varepsilon)}^{2}\right)+\nu \mathbb{D}\left[y_{s, \omega(\varepsilon)}^{1}-y_{s, \omega(\varepsilon)}^{2}\right.
\end{array}\right),
\end{aligned}
$$

where

$$
y_{s, \omega(\varepsilon)}^{i}(\theta) \equiv y^{i}(s+\omega(\varepsilon) \theta), \quad-r \leqq \theta \leqq 0 \quad(i=1,2) .
$$

The linearized equation of $(2.3)$ around $(y(s ; \varepsilon), y(s ; \varepsilon)) \equiv(p(s / \omega(\varepsilon) ; \varepsilon)$, $p(s / \omega(\varepsilon) ; \varepsilon))$ is given by

$$
\left\{\begin{array}{l}
\omega(\varepsilon) \frac{d w^{1}}{d s}(s)=\mathbb{F}_{u}\left(\mu(\varepsilon), y_{s, \omega(\varepsilon)}(\circ ; \varepsilon)\right) w_{s, \omega(\varepsilon)}^{1}+\nu D\left[w_{s, \omega(\varepsilon)}^{2}-w_{s, \omega(\varepsilon)}^{1}\right] \\
\omega(\varepsilon) \frac{d w^{2}}{d s}(s)=\mathbb{F}_{u}\left(\mu(\varepsilon), y_{s, \omega(\varepsilon)}(\circ ; \varepsilon)\right) w_{s, \omega(\varepsilon)}^{2}+\nu D\left[w_{s, \omega(\varepsilon)}^{1}-w_{s, \omega(\varepsilon)}^{2}\right]
\end{array}\right.
$$

By the same transformation as in (1.5), the equation (2.4) is transformed into

$$
\left\{\begin{array}{l}
\omega(\varepsilon) \frac{d}{d s} z^{1}(s)=\mathbb{F}_{u}\left(\mu(\varepsilon), y_{s, \omega(\varepsilon)}(\circ ; \varepsilon)\right) z_{s, \omega(\varepsilon)}^{1}, \\
\omega(\varepsilon) \frac{d}{d s} z^{2}(s)=\left(\mathbb{F}_{u}\left(\mu(\varepsilon), y_{s, \omega(\varepsilon)}(\circ ; \varepsilon)\right)-2 \nu D\right) z_{s, \omega(\varepsilon)}^{2}
\end{array}\right.
$$

The first equation of (2.5) coincides with the linearized equation of (1.2) around the solution $y(s ; \varepsilon)=p(t ; \varepsilon)$, therefore it has the Floquet exponents 0 and $\beta(\varepsilon)$; all 
the rest of Floquet exponents have negative real parts for $\varepsilon \in\left(0, \varepsilon_{0}\right)$.

Next we shall consider the second equation of (2.5). This equation has the same form as the one appearing in $[19 ; \S 3]$. So, applying the result of Lemma $\mathbf{A}$ in $[19$; $\S 3]$ yields the exponents of the second equation of $(2.5)$, for $\nu=\frac{1}{2} \nu_{2} \varepsilon^{2}$, in the following form :

$$
\gamma=\gamma\left(\varepsilon, \nu_{2}\right)=\gamma_{2}\left(\nu_{2}\right) \varepsilon^{2}+\widehat{\gamma}\left(\varepsilon, \nu_{2}\right) \varepsilon^{2}, \hat{\gamma}(0, \cdot)=0
$$

where $\gamma_{2}=\gamma_{2}\left(\nu_{2}\right)$ satisfies

$$
\begin{gathered}
\gamma_{2}^{2}+2 \operatorname{Re}\left(\nu_{2}\left(D \zeta_{1}, \zeta_{0}^{*}\right)-B_{1}\right) \gamma_{2} \\
+\left|\left(D \zeta_{1}, \zeta_{0}^{*}\right)\right|^{2} \nu_{2}^{2}-2 \operatorname{Re}\left(\bar{B}_{1}\left(D \zeta_{1}, \zeta_{0}^{*}\right)\right) \nu_{2}=0, \\
B_{1} \equiv i \omega_{2}-\mu_{2} \operatorname{Re} \frac{d \lambda}{d \mu}(0),
\end{gathered}
$$

$\left(\zeta_{1}, \zeta_{0}^{*}\right.$ are defined in (1.20a) and $\mu_{2}, \omega_{2}$ are as in (2.1)). More precisely, there exists $\varepsilon_{s}, 0<\varepsilon_{s}<\varepsilon_{0}$, such that for each $\varepsilon \in\left(0, \varepsilon_{s}\right)$ and $\mu=\mu(\varepsilon), \nu=\frac{1}{2} \nu_{2} \varepsilon^{2}$ the in-phase solution has the Floquet exponents $\gamma=\gamma\left(\varepsilon, \nu_{2}\right)$ having the form (2.6), where $\nu_{2}$ is not a double root of (2.6). Furthermore we easily see $\gamma\left(\varepsilon, \nu_{2}\right) \rightarrow 0$ or $\beta(\varepsilon)$ as $\nu_{2} \rightarrow 0$.

Thus from the above it follows:

Theorem 1 . Consider the equation (1.1) under the assumptions (A1), (A2) and (A3). If $\operatorname{Re}\left(\overline{B_{1}}\left(D \zeta_{1}, \zeta_{0}^{*}\right)\right)$ in the equation (2.7) is positive (resp. negative), then there exist numbers $\varepsilon_{u}$ and $\nu_{u}$ such that for each $\left(\varepsilon, \nu_{2}\right) \in\left(0, \varepsilon_{u}\right) \times\left(0, \nu_{u}\right), \mu=$ $\mu(\varepsilon)$ and $\nu=\frac{1}{2} \nu_{2} \varepsilon^{2}$ the in-phase solution is unstable (resp. stable).

\section{§3. A Bifrureation of the Amti-PRase Solution}

In the preceding section we have discussed the stability of the in-phase solution which bifurcates from the steady state $\left(x^{1}, x^{2}\right)=(0,0)$. As seen in $\S 1$, in the equation (1.1) another periodic solution bifurcates from the steady state for some parameter values $\mu$ and $\nu$. In this section we shall study the bifurcation of this periodic solution more precisely.

Let $\mu_{0}(\nu)$ and $\alpha_{0}(\nu)$ be functions as in (1.9). Put $\mu=\mu_{0}(\nu)+\mu^{\prime}$. After the change of variables $s=\alpha t, v^{i}(s)=x^{i}(t)(i=1,2),(1.1)$ is transformed into 


$$
\alpha \frac{d}{d s}\left(\begin{array}{c}
v^{1}(s) \\
v^{2}(s)
\end{array}\right)=\left(\begin{array}{l}
F\left(\mu_{0}(\nu)+\mu^{\prime}, v_{s, \alpha}^{1}\right)+\nu D\left[v_{s, \alpha}^{2}-v_{s, \alpha}^{1}\right] \\
F\left(\mu_{0}(\nu)+\mu^{\prime}, v_{s, \alpha}^{2}\right)+\nu D\left[v_{s, \alpha}^{1}-v_{s, \alpha}^{2}\right]
\end{array}\right)
$$

Fix any $\nu \in \mathbb{I}_{\nu}$ and let $\mu^{\prime}$ be a bifurcation parameter, where $\mathbb{I}_{\nu}$ is as in (1.10). If $\alpha_{0}(\nu)$ $\neq \omega_{0}$, then the equation (3.1) satisfies the hypotheses of the Hopf bifurcation theorem at $\mu^{\prime}=0$, and we obtain a periodic solution bifurcating from the steady state. For $\nu=0$ the usual Hopf bifurcation theorem can not apply to (3.1), since the linearized equation (1.3) of (3.1) has double eigenvalues $\pm i \omega_{0}$ at $\mu^{\prime}=\mu=0$, which implies breaking the assumption for the Hopf bifurcation.

In what follows, however, in a specific function space we can construct periodic solutions to (3.1) for $\nu \in \mathbb{I}_{\nu}$ uniformly; then we do not need to assume $\alpha_{0}(\nu) \neq \omega_{0}$. If $\mu_{0}(\nu)(\nu>0)$ is a positive increasing function, we also see from this that for any fixed $\mu=\mu_{0}\left(\nu_{0}\right)\left(\nu_{0} \in(0, \bar{\nu}]\right)$ a periodic solution bifurcating at $\nu=\nu_{0}$ continues to $\nu=0$. As seen in the case of the in-phase solution in $\S 2$, letting $\nu$ continue to decrease to zero, we can see whether or not the stability change occurs. Therefore, the argument mentioned above will be necessary for discussion of the stability change of this periodic solution.

Before introducing the function space, we note that a $2 \pi$-periodic solution to (3.1) which bifurcates at $\mu=\mu_{0}(\nu), \nu \neq 0$, has the form $\left(v^{1}(s), v^{2}(s)\right)=(\phi(s)$, $\phi(s-\pi))$, where $\phi(s)$ is a $2 \pi$-periodic function : In fact, if $\left(v^{1}, v^{2}\right)=\left(\phi^{1}(s), \phi^{2}(s)\right)$ is a $2 \pi$-periodic solution to (3.1), then $\left(\phi^{2}(s), \phi^{1}(s)\right)$ is also so. Uniqueness of the bifurcating solution implies $\left(\phi^{1}(s), \phi^{2}(s)\right)=\left(\phi^{2}(s-l), \phi^{1}(s-l)\right)$ ( $l$ is some number), from which $l=\pi$ follows. Hereafter we call such a periodic solution the anti-phase solution to (3.1) (or (1.1)).

Considering the above property of the anti-phase solution, we shall discuss the bifurcation problem of the anti-phase solution in the space $\mathbb{P}_{2 \pi}^{\prime}$ defined by

$$
\mathbb{P}_{2 \pi}^{\prime}=\left\{w(s) \mid v(s)={ }^{t}(v(s), v(s-\pi)), v \in \mathbb{P}_{2 \pi}\right\},
$$

where $\mathbb{P}_{2 \pi}$ is defined in (1.22a). It is clear that finding a solution to (3.1) in the space $\mathbb{P}_{2 \pi}^{\prime}$ is reduced to finding a solution to the equation

$$
\alpha \frac{d}{d s} v(s)=F\left(\mu_{0}(v)+\mu^{\prime}, v_{s, \alpha}\right)+\nu D\left[v_{s-\pi, \alpha}-v_{s, \alpha}\right], \quad v \in \mathbb{P}_{2 \pi}
$$

Thus we consider the equation (3.2) instead of (3.1).

Let $\tilde{\xi}(\mu, \nu)$ be an eigenvector corresponding to $\tilde{\lambda}(\mu, \nu)$, that is, 


$$
\left(\tilde{\lambda}(\mu, \nu) I-\int_{-r}^{0} e^{\tilde{(}(\mu, \nu) \theta}[d \tilde{\eta}(\theta ; \mu, \nu)]\right) \tilde{\xi}(\mu, \nu)=0,
$$

and suppose that $\tilde{\xi}\left(\mu_{0}(\nu), \nu\right)=\xi_{0}(\nu)$. Differentiating (3.3) with respect to $\mu$ and putting $\mu=\mu_{0}(\nu)$ yield

$$
\begin{aligned}
& \frac{\partial \tilde{\lambda}}{\partial \mu}\left(\mu_{0}(\nu), \nu\right) M(\nu) \xi_{0}(\nu)-\frac{\partial}{\partial \mu} L\left(\mu_{0}(\nu)\right) \xi_{1}(\nu) \\
& \quad+\left(i \alpha_{0}(\nu) I-\int_{-r}^{0} e^{i \alpha_{0}(\nu) \theta}\left[d \tilde{\eta}\left(\theta ; \mu_{0}(\nu), \nu\right)\right]\right) \frac{\partial \tilde{\xi}}{\partial \mu}\left(\mu_{0}(\nu), \nu\right)=0,
\end{aligned}
$$

$(M(\nu)$ is defined in (1.17)). By (1.11b), (1.15) and (1.17) we obtain

$$
\frac{\partial \tilde{\lambda}}{\partial \mu}\left(\mu_{0}(\nu), \nu\right)=\left(F_{\mu u}\left(\mu_{0}(\nu)\right) \xi_{1}(\nu), \xi_{0}^{*}(\nu)\right) .
$$

Next let us define the operator $J(\nu)$ acting in $P_{2 \pi}$ as

$$
\begin{aligned}
& J(\nu) v(s) \equiv \alpha_{0}(\nu) \frac{d}{d s} v(s)-\left(L\left(\mu_{0}(\nu)\right) v_{s, \alpha_{0}}+\nu D\left[v_{s-\pi, \alpha_{0}}-v_{s, \alpha_{0}}\right]\right) \\
& \text { for } v \in \mathscr{D}(J(\nu))\left(=P_{2 \pi}^{(1)}\right),
\end{aligned}
$$

where $\mathfrak{D}(J(\nu))$ denotes the domain of $J(\nu)$. The formal adjoint operator $J^{*}(\nu)$ of $J(\nu)$ is defined by

$$
\left.J^{*}(\nu) v(s) \equiv-\alpha_{0} \frac{d}{d s} v(s)-L^{*}\left(\mu_{0}(\nu)\right) v^{s, \alpha_{0}}+\nu D^{*}\left[v^{s-\pi, \alpha_{0}}-v^{s, \alpha_{0}}\right]\right),
$$

where

$$
\begin{aligned}
& L^{*}(\mu) \psi \equiv \int_{-r}^{0 t}[d \eta(\theta ; \mu)] \psi(-\theta), \quad \psi \in \mathbb{C}[0, r], \\
& D^{*}[\psi] \equiv \int_{-r}^{0 t}\left[d \eta^{\prime}(\theta)\right] \psi(-\theta), \quad \psi \in \mathbb{C}[0, r], \\
& v^{s, \alpha_{0}}(\theta) \risingdotseq v\left(s+\alpha_{0} \theta\right), \quad 0 \leqq \theta \leqq r .
\end{aligned}
$$

For each $\nu \in \mathbb{I}_{\nu}$ the null spaces $\Re(J(\nu))$ and $\mathfrak{R}\left(J^{*}(\nu)\right)$ are spanned by

$$
\begin{array}{cc}
z(\nu) \equiv \xi_{0}(\nu) e^{i s}, & \overline{z(\nu)}, \\
z^{*}(\nu) \equiv \xi_{0}^{*}(\nu) e^{i s}, & \overline{z^{*}(\nu),}
\end{array}
$$

respectively, where $\xi_{0}(\nu)$ and $\xi_{0}^{*}(\nu)$ are as in (1.11).

We define the product in $P_{2 \pi}$ as 


$$
(u, v)_{2 \pi} \equiv \frac{1}{2 \pi} \int_{0}^{2 \pi}(u(s), v(s)) d s, \quad u, v \in \mathbb{P}_{2 \pi}
$$

Then for the operator $J(\nu)$, the next lemma follows from $[7 ;$ Chap. 9]:

Lemma 20 Let $P_{2 \pi}$ be the space defined in (1.22). Consider the equation

$$
J(\nu) u=h, \quad h \in \mathbb{P}_{2 \pi} \quad\left(\nu \in \mathbb{I}_{\nu}\right) .
$$

The equation (3.10) is solvable for $u \in \mathscr{D}(\mathbb{J}(\nu))\left(=\mathbb{P}_{2 \pi}^{(1)}\right)$ if and only if

$$
\left(h, z^{*}(\nu)\right)_{2 \pi}=\left(h, \overline{z^{*}(\nu)}\right)_{2 \pi}=0 \text {. }
$$

Furthermore, there is a continuous projection $\mathbb{P}_{N}(\nu): \mathbb{P}_{2 \pi} \rightarrow \mathbb{P}_{2 \pi}$ such that $\Re(J(\nu))$ $=\left(\mathbb{I}-\mathbb{P}_{N}(\nu)\right) \mathbb{P}_{2 \pi}$ and there is a continuous linear operator $K(\nu):\left(I-P_{N}(\nu)\right) P_{2 \pi}$ $\rightarrow(I-\mathbb{I}) P_{2 \pi} \cap \mathfrak{D}(J(\nu))$ such that $\mathbb{K}(\nu) h$ is a solution of (3.10) for each $h \in$ $\left(I-P_{N}(\nu)\right) P_{2 \pi}$, where $\mathbb{I}(\nu)$ is a continuous projection of $P_{2 \pi}$ onto $\mathfrak{R}(J(\nu))$; moreover $\mathbb{I}(\nu), \mathbb{P}_{N}(\nu)$ and $\mathbb{K}(\nu)$ are smooth in $\nu \in \mathbb{I}_{\nu}$.

By Lemma 2 we obtain the proposition about the bifurcation of the equation (3.2) as follows:

$\mathbb{P r}_{\mathrm{r}} \mathbb{0}$ position 3. Consider the equation (3.2) under the assumptions $(\mathbb{A} 1),(\mathbb{A} 2)$ and (A3). Let the interval $\mathbb{I}_{\nu}$ be as in (1.10). Then for each $\nu \in \mathbb{I}_{\nu}$ a periodic solution bifurcates from the steady state $v=0$ at $\mu^{\prime}=0$ : More precisely, there exists a number $\bar{\varepsilon}>0$, and there exists functions $\mu^{\prime}=\mu^{\prime}(\varepsilon, \nu), \alpha=\alpha(\varepsilon, \nu)$,

$$
\left\{\begin{array}{l}
\mu^{\prime}(\varepsilon, \nu)=\mu_{2}^{\prime}(\nu) \varepsilon^{2}+\widehat{\mu}^{\prime}(\varepsilon, \nu) \varepsilon^{2}, \quad \widehat{\mu}^{\prime}\left(0,{ }^{\circ}\right)=0, \\
\alpha(\varepsilon, \nu)=\alpha_{0}(\nu)+\alpha_{2}(\nu) \varepsilon^{2}+\widehat{\alpha}(\varepsilon, \nu) \varepsilon^{2}, \quad \widehat{\alpha}\left(0,{ }^{\circ}\right)=0,
\end{array}\right.
$$

such that for each $\nu \in I_{\nu}, \varepsilon \in(0, \bar{\varepsilon})$ and $\mu=\mu_{0}(\nu)+\mu^{\prime}(\varepsilon, \nu)$ there is a periodic solution $v(s ; \varepsilon, \nu)$ with period $2 \pi$, where $\mu_{0}(\nu)$ and $\alpha_{0}(\nu)$ are as in (1.9).

Corollary A. Consider the equation (1.1) (or (3.1)) under the same hypotheses in Proposition 3. Then (1.1) has a periodic solution (called the anti-phase solution) bifurcating at $\left(\mu_{0}(\nu), \nu\right), \nu \in \mathbb{I}_{\nu}$. This periodic solution has period $T=2 \pi / \alpha(\varepsilon, \nu)$ and takes the form $\left(x^{1}(t), x^{2}(t)\right)=(v(\alpha t ; \varepsilon, \nu), v(\alpha(t-T / 2) ; \varepsilon, \nu))$ for $(\varepsilon, \nu)$ $\in(0, \bar{\varepsilon}) \times \mathbb{I}_{\nu}$, where $\alpha=\alpha(\varepsilon, \nu)$ and $v(s ; \varepsilon, \nu)$ are as in Proposition 3.

By using arguments analogous to those in $[19 ; \S 2]$ and using the relations 
(1.17), (3.4), the coefficients of lower order terms in $\varepsilon$ of $\mu^{\prime}(\varepsilon, \nu), \alpha(\varepsilon, \nu)$ and $v(s$; $\varepsilon, \nu)$ in Proposition 3 are easily calculated. Considering (1.20) and (1.21), for sufficiently small $\nu$ we obtain

$$
\begin{aligned}
& \left\{\begin{array}{l}
v(s ; \varepsilon, \nu)=v_{1}(s ; \nu) \varepsilon+v_{2}(s ; \nu) \varepsilon^{2}+\widehat{v}(s ; \varepsilon, \nu) \varepsilon^{2}, \\
v_{1}(s ; \nu)=y_{1}(s)+o(\nu), y_{1}(s) \equiv \zeta_{0} e^{i s}+\overline{\zeta_{0}} e^{-i s}, \\
v_{2}(s ; \nu)=y_{2}(s)+o(\nu), y_{2}(s) \equiv \zeta_{2,0} e^{2 i s}+\overline{\zeta_{2,0}} e^{-2 i s}+\bar{\zeta}_{2,0}, \\
\hat{v}(\bullet ; 0, \circ)=0,
\end{array}\right. \\
& \quad \mu_{2}^{\prime}(\nu)=\mu_{2}+o(\nu), \quad \alpha_{2}(\nu)=\omega_{2}+o(\nu), \\
& B_{1} \equiv i \omega_{2}-\mu_{2} \frac{d \lambda}{d \mu}(0) \\
& =\left(F_{u u}(0)\left(\zeta_{1}, \bar{\zeta}_{2}\right), \zeta_{0}^{*}\right) \\
& \quad+\left(F_{u u}(0)\left(\bar{\zeta}_{1}, \zeta_{2}\right), \zeta_{0}^{*}\right)+\frac{1}{2}\left(F_{u u u}(0)\left(\zeta_{1}, \zeta_{1}, \bar{\zeta}_{1}\right), \zeta_{0}^{*}\right),
\end{aligned}
$$

under the condition

$$
\left[v_{s, \alpha_{0}}, z^{* s, \alpha_{0}}(\nu)\right]_{\nu} \equiv \frac{1}{2 \pi} \int_{0}^{2 \pi}\left\langle v_{s, \alpha_{0}}, z^{* s, \alpha_{0}}(\nu)\right\rangle_{\nu}=\varepsilon
$$

where $\zeta_{2,0}, \widehat{\zeta}_{2,0}, \zeta_{2}$ and $\widehat{\zeta}_{2}$ are defined in (1.20b).

\section{§4. On the Stability of the Anti-Phase Solution}

In this section we shall discuss the stability of the anti-phase solution to (3.1) (or (1.1)) by using the same technique as in $[19 ; \S 3]$. The linearized equation of (3.1) around the anti-phase solution is given by

$$
\begin{aligned}
& \alpha(\varepsilon, \nu)\left(\begin{array}{c}
\frac{d w^{1}(s)}{d s} \\
\frac{d w^{2}(s)}{d s}
\end{array}\right) \\
& =\left(\begin{array}{l}
F_{u}\left(\mu_{0}(\nu)+\mu^{\prime}(\varepsilon, \nu), v_{s, \alpha(\varepsilon, \nu)}(\cdot ; \varepsilon, \nu)\right) w_{s, \alpha(\varepsilon, \nu)}^{1}+\nu D\left[w_{s, \alpha(\varepsilon, \nu)}^{2}-w_{s, \alpha(\varepsilon, \nu)}^{1}\right] \\
F_{u}\left(\mu_{0}(\nu)+\mu^{\prime}(\varepsilon, \nu), v_{s-\pi, \alpha(\varepsilon, \nu)}(\cdot ; \varepsilon, \nu)\right) w_{s, \alpha(\varepsilon, \nu)}^{2}+\nu D\left[w_{s, \alpha(\varepsilon, \nu)}^{1}-w_{s, \alpha(\varepsilon, \nu)}^{2}\right]
\end{array}\right),
\end{aligned}
$$

where $\mu^{\prime}(\varepsilon, \nu), \alpha(\varepsilon, \nu)$ and $v(s ; \varepsilon, \nu)$ are as in (3.12) and (3.13). Hereafter, for simplicity of notation, we shall omit $\nu$ as long as there is no confusion.

To determine a Floquet exponent $\gamma$ of (4.1), we have to find a solution to (4.1) 
in the form

$$
\left(\begin{array}{l}
w^{1}(s) \\
w^{2}(s)
\end{array}\right)=e^{\gamma s \mid \omega(\varepsilon)}\left\{p(s)\left(\begin{array}{l}
1 \\
1
\end{array}\right)+q(s)\left(\begin{array}{r}
1 \\
-1
\end{array}\right)\right\}, \quad p, q \in \mathbb{P}_{2 \pi} .
$$

Substituting (4.2) into (4.1) yields the equation

$$
\begin{aligned}
\alpha(\varepsilon) & \frac{d}{d s}\left(\begin{array}{l}
p(s) \\
q(s)
\end{array}\right)=-\gamma\left(\begin{array}{l}
p(s) \\
q(s)
\end{array}\right)-2 \nu\left(\begin{array}{c}
0 \\
D\left[q_{s, \alpha(\varepsilon)} e^{\alpha(\varepsilon) \cdot}\right]
\end{array}\right) \\
& +\left(\begin{array}{l}
\Gamma^{+}(s ; \varepsilon, \nu)\left(p_{s, \alpha(\varepsilon)} e^{\alpha(\varepsilon) \cdot}\right)+\Gamma^{-}(s ; \varepsilon, \nu)\left(q_{s, \alpha(\varepsilon)} e^{\alpha(\varepsilon) \cdot}\right) \\
\Gamma^{-}(s ; \varepsilon, \nu)\left(p_{s, \alpha(\varepsilon)} e^{\alpha(\varepsilon) \cdot}\right)+\Gamma^{+}(s ; \varepsilon, \nu)\left(q_{s, \alpha(\varepsilon)} e^{\alpha(\varepsilon) \cdot}\right)
\end{array}\right),
\end{aligned}
$$

where

$$
\begin{aligned}
& \Gamma^{+}(s ; \varepsilon, \nu) \equiv \frac{1}{2}\left\{F_{u}\left(\mu_{0}+\mu^{\prime}(\varepsilon), v_{s, \alpha(\varepsilon)}(\circ ; \varepsilon)\right)+F_{u}\left(\mu_{0}+\mu^{\prime}(\varepsilon), v_{s-\pi, \alpha(\varepsilon)}(\circ ; \varepsilon)\right)\right\}, \\
& \Gamma^{-}(s ; \varepsilon, \nu) \equiv \frac{1}{2}\left\{F_{u}\left(\mu_{0}+\mu^{\prime}(\varepsilon), v_{s, \alpha(\varepsilon)}(\circ ; \varepsilon)\right)-F_{u}\left(\mu_{0}+\mu^{\prime}(\varepsilon), v_{s-\pi, \alpha(\varepsilon)}(\circ ; \varepsilon)\right)\right\} .
\end{aligned}
$$

As seen in the calculation of the Floquet exponent of the in-phase solution, after the scaling $2 \nu=\nu_{2} \varepsilon^{2}$, we shall seek $\gamma$ which is of order $O\left(\varepsilon^{2}\right)$.

First we shall introduce the function spaces $\mathbb{P}_{2 \pi} \equiv P_{2 \pi} \times P_{2 \pi}$ and $\mathbb{P}_{2 \pi}^{(1)} \equiv P_{2 \pi}^{(1)} \times$ $P_{2 \pi}^{(1)}$, where $P_{2 \pi}$ and $P_{2 \pi}^{(1)}$ are defined in (1.22). $\mathbb{P}_{2 \pi}$ and $\mathbb{P}_{2 \pi}^{(1)}$ are $B$ anach spaces with norms $\left\|{ }^{t}\left(v^{1}, v^{2}\right)\right\| \equiv\left\|v^{1}\right\|+\left\|v^{2}\right\|,{ }^{t}\left(v^{1}, v^{2}\right) \in \mathbb{P}_{2 \pi}$ and $\left\|{ }^{t}\left(v^{1}, v^{2}\right)\right\| \equiv\left\|v^{1}\right\|\|+\| v^{2} \|$, ${ }^{t}\left(v^{1}, v^{2}\right) \in \mathbb{P}_{2 \pi}^{(1)}$ respectively. We difine the product in $\mathbb{P}_{2 \pi}$ as

$$
\begin{aligned}
& (\mathbb{U}, \mathbb{v})_{2 \pi} \equiv\left(u^{1}, v^{1}\right)_{2 \pi}+\left(u^{2}, v^{2}\right)_{2 \pi} \\
& \quad \text { for } u^{t}={ }^{t}\left(u^{1}, u^{2}\right), \mathbb{v}={ }^{t}\left(v^{1}, v^{2}\right) \in \mathbb{P}_{2 \pi} .
\end{aligned}
$$

Next let us define the operator $\mathbb{J}_{0}$ acting in $\mathbb{P}_{2 \pi}$ as

$$
\mathbb{J}_{0} Q(s) \equiv\left(\begin{array}{l}
J(0) p(s) \\
J(0) q(s)
\end{array}\right), \quad Q={ }^{t}(p, q) \in \mathbb{P}_{2 \pi}^{(1)}
$$

where $J(\nu)$ is defined by (3.5). It is clear that the null space of $\mathscr{J}_{0}$ is spanned by the elements $z_{i}, \bar{z}_{i}(i=1,2)$,

$$
z_{1} \sum^{t}(z(0), 0), \quad z_{2} \bar{\aleph}^{t}(0, z(0)) \quad(z(\nu) \text { is as in (3.7)). }
$$

We define the formal adjoint operator $\mathscr{J}_{0}^{*}$ by 


$$
\mathscr{J}_{0}^{* *} Q(s) \equiv\left(\begin{array}{l}
J^{*}(0) p(s) \\
J^{*}(0) q(s)
\end{array}\right), \quad Q={ }^{t}(p, q) \in \mathbb{P}_{2 \pi}^{(1)}
$$

By the definition of $\mathscr{H}_{0}^{*}$,

$$
\begin{array}{ll}
\mathbb{J}_{0}^{*} z_{i}^{*}=\mathbb{J}_{0}^{*} z_{i}^{*}=0 & (i=1,2), \\
z_{1}^{*} \bar{F}^{t}\left(z^{*}(0), 0\right), & z_{2}^{*} \bar{F}^{t}\left(0, z^{*}(0)\right) \quad\left(z^{*}(\nu) \text { is as in }(3.8)\right) .
\end{array}
$$

Furthermore, let

$$
\mathbb{P}_{N} \equiv \mathbb{P}_{N}(0) \times \mathbb{P}_{N}(0), \quad \mathbb{K} \equiv \mathbb{K}(0) \times \mathbb{K}(0), \quad \mathbb{I I} \equiv \mathbb{I}(0) \times \mathbb{I}(0)
$$

where $P_{N}(\nu), K(\nu)$ and $\Pi(\nu)$ are the operators defined in Lemma 2 . Then for the above operator $\mathbb{J}_{0}$ Fredholm alternative holds in the space $\mathbb{P}_{2 \pi}$ by Lemma 2 ; that is, the equation

$$
\mathbb{J}_{0}=\mathbb{R}, \quad R \in \mathbb{P}_{2 \pi}
$$

is solvable for $\mathbb{u} \in \mathfrak{D}\left(\mathbb{J}_{0}\right)\left(=\mathbb{P}_{2 \pi}^{(1)}\right)$, if and only if

$$
\left(\mathbb{R}, z_{i}^{*}\right)_{2 \pi}=\left(\mathbb{R}, \overline{z_{i}^{*}}\right)_{2 \pi}=0 \quad(i=1,2) .
$$

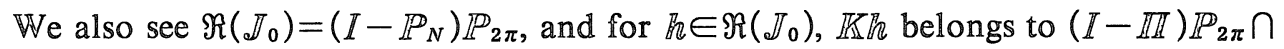
$\mathfrak{D}\left(\mathbb{J}_{0}\right)$.

Now we shall find the solution to (4.3) in the form

$$
\left\{\begin{array}{l}
2 \nu=\nu_{2} \varepsilon^{2}, \\
\gamma(\varepsilon)=\gamma_{2} \varepsilon^{2}+\hat{\gamma}(\varepsilon) \varepsilon^{2}, \quad \hat{\gamma}(0)=0, \\
Q(s ; \varepsilon)=Q_{0}(s ; \varepsilon)+Q_{1}(s ; \varepsilon) \varepsilon+\hat{Q}(s ; \varepsilon) \varepsilon, \\
\widehat{Q}, Q_{i} \in \mathbb{P}_{2 \pi}^{(1)}(i=0,1), Q_{i}(s ; 0) \neq 0(i=0,1), \hat{Q}(s ; 0) \equiv 0,
\end{array}\right.
$$

under the condition

$$
\begin{aligned}
& \llbracket Q_{s, \omega_{0},} z_{i}^{* s, \omega_{0}} \rrbracket=c_{i}+\eta_{i}(\varepsilon), \quad \llbracket Q_{s, \omega_{0}}, \overline{z_{i}^{* s, \omega_{0}}} \rrbracket=c_{i}^{\prime}+\eta_{i}^{\prime}(\varepsilon), \\
& \eta_{i}(0)=\eta_{i}^{\prime}(0)=0 \quad(i=1,2),
\end{aligned}
$$

where the notation $\llbracket{ }^{\circ}, \circ \rrbracket$ is defined as

$$
\begin{aligned}
& \left.\llbracket P_{s, \omega_{0}}, U^{s, \omega_{0}}\right] \equiv\left[p_{s, \omega_{0}}, u^{s, \omega_{0}}\right]_{0}+\left[q_{s, \omega_{0}}, v^{s, \omega_{0}}\right]_{0}, \\
& \quad P={ }^{t}(p, q), U={ }^{t}(u, v) \in \mathbb{P}_{2 \pi} \quad\left(\left[{ }^{\circ},\right]_{\nu}\right. \text { is as in (3.16)). }
\end{aligned}
$$


Note that in general $Q$ and $\gamma$ will be obtained as complex valued functions in $\varepsilon$, and that from (3.12) and (3.14) we see that

$$
\begin{aligned}
& \mu_{0}(\nu)+\mu^{\prime}(\varepsilon, \nu)=\left(\frac{1}{2} \nu_{2} \frac{d \mu_{0}}{d \nu}(0)+\mu_{2}\right) \varepsilon^{2}+O\left(\varepsilon^{3}\right), \\
& \alpha(\varepsilon, \nu)=\omega_{0}+\left(\frac{1}{2} \nu_{2} \frac{d \alpha_{0}}{d \nu}(0)+\omega_{2}\right) \varepsilon^{2}+O\left(\varepsilon^{3}\right)
\end{aligned}
$$

hold for $\nu$ in (4.9).

Substituting (4.9) into (4.3) and (4.10) yields the following equations, from which we get the coefficients $Q_{0}, Q_{1}$ and $\widehat{Q}$ iteratively:

$$
\begin{gathered}
\mathbb{J}_{0} Q_{0}=0, \quad \llbracket Q_{0}, z_{i}^{*} \rrbracket=c_{i}+\eta_{i}, \llbracket Q_{0}, \overline{z_{i}^{*} \rrbracket} \rrbracket=c_{i}^{\prime}+\eta_{i}^{\prime}(i=1,2), \\
\mathbb{J}_{0} Q_{1}(s)=\left(\begin{array}{c}
F_{u u}(0)\left(v_{1, s, w_{0}}(s), q_{0}(s)\right) \\
F_{u u}(0)\left(v_{1, s, \omega_{0}}(s), p_{0}(s)\right)
\end{array}\right), \\
\llbracket Q_{1}, z_{i}^{*} \rrbracket=\llbracket Q_{1}, \overline{z_{i}^{*} \rrbracket} \rrbracket=0 \quad(i=1,2), \\
\mathbb{J}_{0} \hat{Q}=R\left(\varepsilon, \hat{\gamma}, \eta_{1}, \eta_{1}^{\prime}, \eta_{2}, \eta_{2}^{\prime}, \hat{Q}\right), \\
\llbracket \hat{Q}, z_{i}^{*} \rrbracket=\llbracket \hat{Q}, \overline{z_{i}^{*}} \rrbracket=0 \quad(i=1,2),
\end{gathered}
$$

where the remaining term $R$ can be expressed as

$$
\begin{aligned}
& R\left(\varepsilon, \hat{\gamma}, \eta_{1}, \eta_{1}^{\prime}, \eta_{2}, \eta_{2}^{\prime}, \widehat{Q}\right)=R_{1}\left(\varepsilon, \hat{\gamma}, \eta_{1}, \eta_{1}^{\prime}, \eta_{2}, \eta_{2}^{\prime}, \hat{Q}\right) \\
& +\varepsilon R_{2}\left(\varepsilon, \hat{\gamma}, \eta_{1}, \eta_{1}^{\prime}, \eta_{2}, \eta_{2}^{\prime}, \hat{Q}\right) \text {, } \\
& R_{1}\left(\varepsilon, \hat{\gamma}, \eta_{1}, \eta_{1}^{\prime}, \eta_{2}, \eta_{2}^{\prime}, \widehat{Q}\right)(s)=L(0)\left(\widehat{Q}_{s, \alpha(\varepsilon, \nu)}-\widehat{Q}_{s, \omega_{0}}\right) \\
& +\varepsilon\left[-\left(\frac{1}{2} \nu_{2} \frac{d \alpha_{0}}{d \nu}(0)+\omega_{2}\right)\left\{\frac{d}{d s} Q_{0}(s)-\mathbb{L}(0)\left(\cdot \frac{d}{d s} Q_{0, s, \omega_{0}}(\cdot)\right)\right\}\right. \\
& -\left(\gamma_{2}+\hat{\gamma}\right)\left\{Q_{0}(s)-\mathbb{L}(0)\left(\cdot Q_{0, s, \omega_{0}}(\cdot)\right)\right\} \\
& +\left(\frac{1}{2} \nu_{2} \frac{d \mu_{0}}{d \nu}(0)+\mu_{2}\right) \dot{F}_{\mu u}(0) Q_{0, s, \omega_{0}}-\nu_{2}\left(\begin{array}{c}
0 \\
D\left[q_{0, s, \omega_{0}}\right]
\end{array}\right)
\end{aligned}
$$

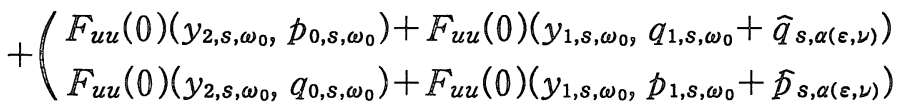

$$
\begin{aligned}
& \begin{array}{l}
\left.\left.+\frac{1}{2} \mathbb{F}_{u u u}(0)\left(y_{1, s, \omega_{0}}, p_{0, s, \omega_{0}}, p_{0, s, \omega_{0}}\right)\right)\right], \\
+\frac{1}{2} \mathbb{F}_{u u u}(0)\left(y_{1, s, \omega_{0}}, q_{0, s, \omega_{0}}, q_{0, s, \omega_{0}}\right)
\end{array} \\
& \mathbb{R}_{\mathbf{2}}\left(0,{ }^{\circ},{ }^{\circ},{ }^{\circ},{ }^{\circ},{ }^{\circ}\right)(s) \equiv 0 \text {, }
\end{aligned}
$$


where $y_{1}, y_{2}$ are as in (3.13), and note that $y_{1}(s-\pi)=-y_{1}(s), y_{2}(s-\pi)=y_{2}(s)$.

Solving (4.11) and (4.12) in $Q_{0}$ and $Q_{1}$ gives

$$
\begin{aligned}
Q_{0}(s)=\left(c_{1}+\eta_{1}\right) z_{1}+\left(c_{1}^{\prime}+\eta_{1}^{\prime}\right) \bar{z}_{1}+\left(c_{2}+\eta_{2}\right) z_{2}+\left(c_{2}^{\prime}+\eta_{2}^{\prime}\right) \bar{z}_{2} \\
\quad\left(c_{1}, c_{1}^{\prime}, c_{2}, c_{2}^{\prime}\right) \neq 0 \\
Q_{1}(s)=2\left(\begin{array}{c}
c_{2}+\eta_{2} \\
c_{1}+\eta_{1}
\end{array}\right) \zeta_{2,0} e^{2 i s}+2\left(\begin{array}{l}
c_{2}^{\prime}+\eta_{2}^{\prime} \\
c_{1}^{\prime}+\eta_{1}^{\prime}
\end{array}\right) \overline{\zeta_{2,0}} e^{-2 i s} \\
+\left(\begin{array}{c}
c_{2}+c_{2}^{\prime}+\eta_{2}+\eta_{2}^{\prime} \\
c_{1}+c_{1}^{\prime}+\eta_{1}+\eta_{1}^{\prime}
\end{array}\right) \bar{\zeta}_{2,0}
\end{aligned}
$$

$\left(\zeta_{2,0}, \widehat{\zeta}_{2,0}\right.$ are defined in $\left.(1.20 b)\right)$.

Finally we shall solve the equation (4.13). Using the operator $\mathbb{R}$ and $\mathbb{P}_{N}$ in (4.8), we get the equations which are equivalent to (4.13),

$$
\left\{\begin{array}{l}
\hat{Q}=\mathbb{K}\left(I-\mathbb{P}_{N}\right) R\left(\varepsilon, \hat{\gamma}, \eta_{1}, \eta_{1}^{\prime}, \eta_{2}, \eta_{2}^{\prime}, \hat{Q}\right), \quad \hat{Q} \in \mathbb{P}_{2 \pi}^{(1)} \\
\mathbb{P}_{N} R\left(\varepsilon, \hat{\gamma}, \eta_{1}, \eta_{1}^{\prime}, \eta_{2}, \eta_{2}^{\prime}, \widehat{Q}\right)=0 .
\end{array}\right.
$$

By applying the implicit function theorem to the first equation of (4.16), we get the solution $\hat{Q}=\widehat{Q}^{*}\left(\varepsilon, \hat{\gamma}, \eta_{1}, \eta_{1}^{\prime}, \eta_{2}, \eta_{2}^{\prime}\right)\left(\in \mathbb{P}_{2 \pi}^{(1)}\right)$ which is smooth in its arguments and satisfies $\hat{Q}^{*}=\frac{\partial \hat{Q}^{*}}{\partial \hat{\gamma}}=\frac{\partial \hat{Q}^{*}}{\partial \eta_{i}}=\frac{\partial \hat{Q}^{*}}{\partial \eta_{i}^{\prime}}=0(i=1,2)$ for $\varepsilon=0$. Substituting $\hat{Q}^{*}$ into the second equation of (4.16) yields

$$
\mathbb{P}_{N} R\left(\varepsilon, \hat{\gamma}, \eta_{1}, \eta_{1}^{\prime}, \eta_{2}, \eta_{2}^{\prime}, \hat{Q}^{*}\left(\varepsilon, \hat{\gamma}, \eta_{1}, \eta_{1}^{\prime}, \eta_{2}, \eta_{2}^{\prime}\right)\right)=0
$$

which implies $\left(R, z_{i}^{*}\right)_{2 \pi}=\left(R, \overline{z_{i}^{*}}\right)_{2 \pi}=0(i=1,2)$. Let

$$
\left\{\begin{aligned}
W_{i}^{(1)} & \left(\varepsilon, \hat{\gamma}, \eta_{1}, \eta_{1}^{\prime}, \eta_{2}, \eta_{2}^{\prime}\right) \\
& \equiv \frac{1}{\varepsilon}\left(R\left(\varepsilon, \hat{\gamma}, \eta_{1}, \eta_{1}^{\prime}, \eta_{2}, \eta_{2}^{\prime}, \widehat{Q}^{*}\right), z_{i}^{*}\right)_{2 \pi}=0 \quad(i=1,2), \\
W_{i}^{(2)} & \left(\varepsilon, \hat{\gamma}, \eta_{1}, \eta_{1}^{\prime}, \eta_{2}, \eta_{2}^{\prime}\right) \\
& \equiv \frac{1}{\varepsilon}\left(R\left(\varepsilon, \hat{\gamma}, \eta_{1}, \eta_{1}^{\prime}, \eta_{2}, \eta_{2}^{\prime}, \widehat{Q}^{*}\right), \overline{z_{i}^{*}}\right)_{2 \pi}=0 \quad(i=1,2) .
\end{aligned}\right.
$$

After a little tedious calculation, using the relations (1.15)-(1.17), (3.4) and $(\alpha 6),(\alpha 7)$ in Appendix, one will see that $W_{i}^{(j)}(0,0,0,0,0,0)=0(i, j=1,2)$ hold if and only if

$$
\left(-\gamma_{2}+\nu_{2}\left(D \zeta_{1}, \zeta_{0}^{*}\right)+B_{1}\right) c_{1}+B_{1} c_{1}^{\prime}=0
$$




$$
\begin{aligned}
& \left(-\gamma_{2}+\nu_{2}\left(D \bar{\zeta}_{1}, \overline{\zeta_{0}^{*}}\right)+\bar{B}_{1}\right) c_{1}^{\prime}+\bar{B}_{1} c_{1}=0 \\
& \left(-\gamma_{2}+B_{1}\right) c_{2}+B_{1} c_{2}^{\prime}=0 \\
& \left(-\gamma_{2}+\bar{B}_{1}\right) c_{2}^{\prime}+\bar{B}_{1} c_{2}=0
\end{aligned}
$$

hold, where $B_{1}$ is given by (2.7b) and (3.15). Thus, if $\gamma_{2}$ satisfies the equation

$$
\begin{aligned}
\gamma_{2}^{2}- & 2 \operatorname{Re}\left(\nu_{2}\left(D \zeta_{1}, \zeta_{0}^{*}\right)+B_{1}\right) \gamma_{2} \\
& +\left|\left(D \zeta_{1}, \zeta_{0}^{*}\right)\right|^{2} \nu_{2}^{2}+2 \operatorname{Re}\left(\bar{B}_{1}\left(D \zeta_{1}, \zeta_{0}^{* *}\right)\right) \nu_{2}=0
\end{aligned}
$$

then there exists $\left(c_{1}, c_{1}^{\prime}, c_{2}, c_{2}^{\prime}\right)$ satisfying (4.18) and (4.19) such that $\left(c_{1}, c_{1}^{\prime}\right) \neq 0$, $c_{2}=c_{2}^{\prime}=0$. And if $\gamma_{2}$ satisfies the equation

$$
\gamma_{2}^{2}-\left(2 \operatorname{Re} B_{1}\right) \gamma_{2}=0
$$

that is, $\gamma_{2}=0$ or $\beta_{2}$, then there exists $\left(c_{1}, c_{1}^{\prime}, c_{2}, c_{2}^{\prime}\right)$ satisfying (4.18) and (4.19) such that $c_{1}=c_{1}^{\prime}=0,\left(c_{2}, c_{2}^{\prime}\right) \neq 0$, where $\beta_{2}$ is as in (2.2).

Under the condition (4.20) or (4.21) for $\gamma_{2}$, applying the implicit function theorem to the equation (4.17) yields the following lemma :

Lemman 5. Consider the linearized equation (4.1) around the anti-phase solution to (1.1) under the hypotheses in Theorem 1. Let $\gamma_{2}=\gamma_{2}\left(\nu_{2}\right)$ be a root of the equation (4.20). Suppose that $\gamma_{2}\left(\nu_{2}\right) \neq 0, \beta_{2}$ and that $\gamma_{2}\left(\nu_{2}\right)$ is not a double root of (4.20). Then there exists a positive constant $\varepsilon_{\delta}$ depending on $\nu_{2}$, such that for each $\varepsilon \in\left(0, \varepsilon_{\delta}\right), \nu=\frac{1}{2} \nu_{2} \varepsilon^{2}$ and $\mu=\mu_{0}(\nu)+\mu^{\prime}(\varepsilon, \nu)$ the equation (4.1) has the Floquet exponents $0, \beta(\varepsilon)$ and $\gamma=\gamma\left(\varepsilon, \nu_{2}\right)=\gamma_{2}\left(\nu_{2}\right) \varepsilon^{2}+\hat{\gamma}\left(\varepsilon, \nu_{2}\right) \varepsilon^{2}\left(\hat{\gamma}\left(0,{ }^{\circ}\right)=0\right)$, where $\beta(\varepsilon)$ and $\beta_{2}$ are as in (2.2). Furthermore $\gamma\left(\varepsilon,{ }^{\circ}\right)$ is smooth in $\varepsilon$.

The proof of Lemma 5 will be left to Appendix $\mathbb{B}$. The next theorem immediately follows from the above lemma :

Theorem 6. Suppose that the hypotheses in Lemma 5 hold. If $\operatorname{Re}\left(\bar{B}_{1}\left(D \zeta_{1}\right.\right.$, $\left.\zeta_{0}^{*}\right)$ ) is positive (resp. negative), then for sufficiently small $\nu$ there exists a value $\mu$ for which the anti-phase solution is stable (resp. unstable), where $B_{1}$ is given by (3.15).

Now, if $\operatorname{Re}\left(D \zeta_{1}, \zeta_{0}^{*}\right)>0$ and $\operatorname{Re} \frac{d \lambda}{d \mu}(0)>0$, then $\mu_{0}(\nu)>0$ for sufficiently small $\nu>0$ (see $(\alpha 6)$ in Appendix A). This implies that the in-phase solution (resp. the 
anti-phase solution) of $(2.1)$ is stable (resp. unstable) if we take $\mu$ (resp. $\mu^{\prime}$ ) sufficiently small for a fixed $\nu>0$. Also if $\operatorname{Re}\left(D \zeta_{1}, \zeta_{0}^{*}\right)<0$ and $\operatorname{Re} \frac{d \lambda}{d \mu}(0)<0$, a similar fact holds by replacing the signs in the above. Considering this and the equations (2.7a), (4.20) yields

Corollary 7. Let the hypotheses in Theorem 6 hold and let $\operatorname{Re}\left(D \zeta_{1}, \zeta_{0}^{*}\right) \times$ $\operatorname{Re} \frac{d \lambda}{d \mu}(0)>0, \operatorname{Re}\left(\bar{B}_{1}\left(D \zeta_{1}, \zeta_{0}^{*}\right)\right)>0$. Fix $\nu>0$ sufficiently small. Then the in-phase solution of (2.1) loses its stability for some parameter value $\mu$ while there exists a value $\mu$ for which the anti-phase solution of (1.1) recovers its stability.

Furthermore, from the equation (4.20) it follows :

Corollary 8. Assume the hypotheses in Theorem 6. Let $\operatorname{Re}\left(\bar{B}_{1}\left(D \zeta_{1}, \zeta_{0}^{*}\right)\right)<0$. If $\operatorname{Re} \frac{d \lambda}{d \mu}(0)>0($ resp. $<0)$ and $\frac{\operatorname{Re} B_{1}}{\operatorname{Re}\left(D \zeta_{1}, \zeta_{0}^{*}\right)}<\frac{\operatorname{Re}\left(\bar{B}_{1}\left(D \zeta_{1}, \zeta_{0}^{*}\right)\right)}{\left|\left(D \zeta_{1}, \zeta_{0}^{*}\right)\right|^{2}}$, then there exists pairs $\left(\mu^{k}, \nu\right)(k=1,2,3), \mu^{1}<\mu^{2}<\mu^{3}$ (resp. $\left.\mu^{1}>\mu^{2}>\mu^{3}\right)$, such that the anti-phase solution is stable for $\left(\mu^{1}, \nu\right),\left(\mu^{3}, \nu\right)$ and is unstable for $\left(\mu^{2}, \nu\right)$; this means that the stability change of the anti-phase solution occurs twice at least.

\section{§5. Applications}

In this section we shall apply the results obtained in $\S 2$ and $\S 4$ to some specific equations.

a) First let us consider the following linear difference coupled delay-differential equation:

$$
\left\{\begin{array}{l}
\dot{x}^{1}(t)=\nu\left(x^{2}(t)-x^{1}(t)\right)-\left(\frac{\pi}{2}+\mu\right)\left(1+x^{1}(t-1)\right) x^{1}(t) \\
\dot{x}^{2}(t)=\nu\left(x^{1}(t)-x^{2}(t)\right)-\left(\frac{\pi}{2}+\mu\right)\left(1+x^{2}(t-1)\right) x^{2}(t)
\end{array}\right.
$$

where $\nu \geqq 0$. In the scalar equation,

$$
\dot{x}(t)=-\left(\frac{\pi}{2}+\mu\right)(1+x(t-1)) x(t),
$$

a Hopf bifurcation occurs at $\mu=0$ and its bifurcating periodic solution is stable near the bifurcation point as mentioned in the Introduction ([3], [8]).

The linear part and the nonlinear part of (5.2) are 


$$
\begin{aligned}
& \mathbb{L}(\mu) \phi=-\left(\frac{\pi}{2}+\mu\right) \phi(-1) \\
& G(\mu, \phi)=-\left(\frac{\pi}{2}+\mu\right) \phi(0) \phi(-1)
\end{aligned}
$$

respectively. In the equation (5.1), the operator $D$ in (1.1) is

$$
D[\phi]=\phi(0) \text { for } \phi \in \mathbb{C}[-1,0] .
$$

The bilinear form, in this case, is defined as

$$
\langle\phi, \psi\rangle_{0}=\overline{\psi(0)} \phi(0)-\frac{\pi}{2} \int_{-1}^{0} \overline{\psi(\xi+1)} \phi(\xi) d \xi, \quad \phi \in \mathbb{C}[-1,0], \phi \in \mathbb{C}[0,1]
$$

$\zeta_{1}, \zeta_{1}^{*}$ and $B_{1}$ in (1.20a) and (3.15) are easily calculated as

$$
\begin{aligned}
& \zeta_{1}(\theta)=e^{i \frac{\pi}{2} \theta}, \quad-1 \leqq \theta \leqq 0 \quad\left(\zeta_{0}=1\right), \\
& \zeta_{1}^{*}(\theta)=\varkappa e^{i \frac{\pi}{2} \theta}, \quad 0 \leqq \theta \leqq 1 \quad\left(\zeta_{0}^{*}=\varkappa\right), \varkappa=\frac{1}{1-\frac{\pi}{2} i}, \\
& B_{1}=\frac{\pi}{10}(1-3 i) \bar{\varkappa},
\end{aligned}
$$

(see $[19 ; \S 5])$. Hence we get

$$
\left(D \zeta_{1}, \zeta_{0}^{*}\right)=\bar{x}, \quad \operatorname{Re}\left(\bar{B}_{1}\left(D \zeta_{1}, \zeta_{0}^{*}\right)\right)=\frac{\pi}{10}|x|^{2}>0
$$

which implies that, in the present case, the conditions in Corollary 7 are satisfied.

Next, in the $(\mu, \nu)$-plane, we shall calculate slopes, at the origin, of the curves $l_{1}$ and $l_{2}$, in which the stability changes of the in-phase and the anti-phase solutions occur respectively. Let $\nu_{2, s}$ be the value $2 \operatorname{Re}\left(\bar{B}_{1}\left(D \zeta_{1}, \zeta_{0}^{*}\right)\right) /\left|\left(D \zeta_{1}, \zeta_{0}^{*}\right)\right|^{2}=\frac{\pi}{5}$, for which (2.7a) has a zero root, and let $\nu_{2, c}$ be the value $-\operatorname{Re} B_{1} / \operatorname{Re}\left(D \zeta_{1}, \zeta_{0}^{*}\right)=$ $\frac{\pi(3 \pi-2)}{20}$, for which (4.20) has a pair of pure imaginary roots. $\mu(\varepsilon)$ in $(2.1)$, in this case, is given as $\mu(\varepsilon)=\frac{3 \pi-2}{10} \varepsilon^{2}+O\left(\varepsilon^{3}\right)$; from which the slope of $l_{1}$ at $(\mu, \nu)=$ $(0,0)$ is given by $\left(\frac{\frac{1}{2} \nu_{2, s} \varepsilon^{2}}{\mu(\varepsilon)}\right)_{\varepsilon=0}=\frac{\pi}{3 \pi-2}$. For the value $\nu=\frac{1}{2} \nu_{2, c} \varepsilon^{2}$, we get

$$
\mu=\mu_{0}(\nu)+\mu^{\prime}(\varepsilon, \nu)=\left(\frac{1}{2} \nu_{2, c} \frac{d \mu_{0}}{d \nu}(0)+\mu_{2}\right) \varepsilon^{2}+O\left(\varepsilon^{3}\right)
$$




$$
=\frac{3 \pi-2}{5} \varepsilon^{2}+O\left(\varepsilon^{3}\right)
$$

by (3.12), (3.14) and $(\alpha 6)$ (in Appendix A), so the slope of $l_{2}$ is $\frac{1}{2} \frac{\pi(3 \pi-2)}{20} /$ $\frac{3 \pi-2}{5}=\frac{\pi}{8}$. Furthermore, $1 / \frac{d \mu_{0}}{d \nu}(0)=\frac{\pi}{4}$ gives a slope of a bifurcation curve of the anti-phase solution at the origin $(0,0)$. Thus we obtain Fig. 1 in a sufficiently small neighborhood of the origin.

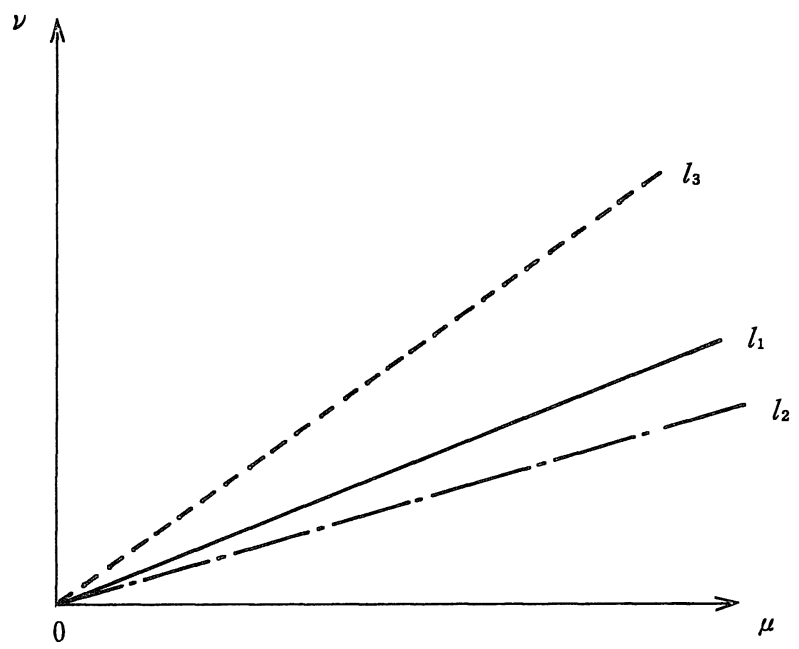

Figure 1. $l_{1}$ and $l_{2}$ are the curves of stability change for the in-phase and the anti-phase solutions respectively. $l_{3}$ is a bifurcation curve of the anti-phase solution.

b) If $F\left(\mu,{ }^{\circ}\right)$ in (1.2) satisfies

$$
F(\mu, \phi)=f(\mu, \phi(0)) \text { for } \phi \in \mathbb{C}[-r, 0] \text {, }
$$

where $f: \mathbb{I}_{0} \times \mathbb{R}^{m} \rightarrow \mathbb{R}^{m}$ is a smooth function, then the equation (1.2) is identified with the ordinary differential equation (without time delay),

$$
\dot{x}(t)=f(\mu, x(t)) \text {. }
$$

Thus, our results obtained in the preceding sections hold for the linear difference 
coupled equation,

$$
\left\{\begin{array}{l}
\dot{x}^{1}(t)=f\left(\mu, x^{1}(t)\right)+\nu D\left(x^{2}(t)-x^{1}(t)\right) \\
\dot{x}^{2}(t)=f\left(\mu, x^{2}(t)\right)+\nu D\left(x^{1}(t)-x^{2}(t)\right)
\end{array}\right.
$$

where $D$ is an $m \times m$ matrix. Note that in this case, $L(\mu)=\frac{\partial}{\partial x} f(\mu, 0),\left\langle\circ,{ }^{\circ}\right\rangle_{\nu}=$ $\left({ }^{\circ},{ }^{\circ}\right)$ and so forth.

Now let us consider a biochemical model, the Brusselator, that is,

$$
\left\{\begin{array}{l}
\dot{x}(t)=A-(B+1) x+x^{2} y \\
\dot{y}(t)=B x-x^{2} y
\end{array}\right.
$$

where $A$ and $B$ are positive constants (see [24]). The equation (5.6) has the steady state $(x(t), y(t)) \equiv\left(A, \frac{B}{A}\right)$. Let $B=A^{2}+1+\mu$. Then in the equation (5.6) a Hopf bifurcation occurs at $\mu=0$ and this bifurcating periodic solution is stable near the bifurcation point.

Using the equation (5.6), we shall consider the equation,

$$
\left\{\begin{array}{l}
\dot{x}^{i}(t)=A-(B+1) x^{i}+\left(x^{i}\right)^{2} y^{i}+\nu d_{1}\left(x^{j}-x^{i}\right), \\
\dot{y}^{i}(t)=B x^{i}-\left(x^{i}\right)^{2} y^{i}+\nu d_{2}\left(y^{j}-y^{i}\right), \quad i, j=1,2, i \neq j,
\end{array}\right.
$$

where $d_{i} \geqq 0(i=1,2)$. After a little tedious calculation, we get $\zeta_{1}$, $\zeta_{1}^{*}$ and $B_{1}$ as

$$
\begin{aligned}
& \zeta_{1}=\left(\begin{array}{c}
1 \\
\frac{i-A}{A}
\end{array}\right), \zeta_{1}^{*}=\varkappa\left(\begin{array}{c}
1 \\
\frac{A}{A-i}
\end{array}\right), \varkappa=\frac{1+A i}{2}, \\
& B_{1}=-\frac{1}{2}\left(\frac{A^{2}+2}{A^{2}}+i \frac{4 A^{4}-7 A^{2}+4}{3 A^{3}}\right) .
\end{aligned}
$$

Hence,

$$
\begin{aligned}
& \left(D \zeta_{1}, \zeta_{0}^{*}\right)=\frac{1}{2}\left\{\left(d_{1}+d_{2}\right)+i A\left(d_{2}-d_{1}\right)\right\} \\
& \operatorname{Re}\left(\bar{B}_{1}\left(D \zeta_{1}, \zeta_{0}^{*}\right)\right)=-\frac{1}{12 A^{2}}\left\{3\left(d_{1}+d_{2}\right)\left(A^{2}+2\right)+\left(d_{2}-d_{1}\right)\left(4 A^{4}-7 A^{2}+4\right)\right\}
\end{aligned}
$$

For simplicity, we put $d_{2}=0$. Then one will easily see that the equation (5.7) satisfies the conditions in Corollary 7 (resp. Corollary 8) for sufficiently large (resp. small) value of $A$. 


\section{Appendix A。}

Let us consider the equation,

$(\alpha 1) \quad \Delta(\omega, \mu, \nu) \xi_{0} \equiv\left(i \omega I-\int_{-r}^{0} e^{i \omega \theta}[d \eta(\theta ; \mu)]+2 \nu \int_{-r}^{0} e^{i \omega \theta}\left[d \eta^{\prime}(\theta)\right]\right) \xi_{0}=0$.

We shall seek smooth functions $\mu=\mu_{0}(\nu), \omega=\alpha_{0}(\nu)$ and $\xi_{0}=\xi_{0}(\nu)$ satisfying $(\alpha 1)$ for small $\nu$. The argument below is along the line found in $[7 ;$ Chap. 7].

For $\mu=\nu=0, \xi_{0}=\zeta_{0}$ and $\omega=\omega_{0}$ satisfy $(\alpha 1)$, that is,

$$
\Delta\left(\omega_{0}, 0,0\right) \zeta_{0}=\left(i \omega_{0} I-\int_{-r}^{0} e^{i \omega_{0} \theta}[d \eta(\theta ; 0)]\right) \zeta_{0}=0 .
$$

The assumption (A1) implies that the null space $\mathfrak{N}\left(\Delta\left(\omega_{0}, 0,0\right)\right)$ has dimension one, and $\mathbb{C}^{m}$ is decomposed as

$$
\mathbb{C}^{m}=\mathfrak{N}\left(\Delta\left(\omega_{0}, 0,0\right)\right) \oplus \Re\left(\Delta\left(\omega_{0}, 0,0\right)\right) .
$$

Since $\zeta_{0}^{*}$ as in (1.20a) belongs to $\mathfrak{N}\left(\overline{\Delta\left(\omega_{0}, 0,0\right)}\right)$,

$$
\Re\left(\Delta\left(\omega_{0}, 0,0\right)\right)=\left\{\xi \mid \xi \in \mathbb{C}^{m},\left(\xi, \zeta_{0}^{*}\right)=0\right\} .
$$

Let $\pi_{0}$ be a projection of $\mathbb{C}^{m}$ onto $\mathfrak{N}\left(\Delta\left(\omega_{0}, 0,0\right)\right)$. Then $(\alpha 1)$ is reduced to the equations,

$$
\begin{gathered}
\Delta\left(\omega_{0}, 0,0\right) \xi_{0}^{R}=\left(I-\pi_{0}\right)\left[\Delta\left(\omega_{0}, 0,0\right)-\Delta(\omega, \mu, \nu)\right]\left(\zeta_{0}+\xi_{0}^{R}\right), \\
\pi_{0}\left[\Delta\left(\omega_{0}, 0,0\right)-\Delta(\omega, \mu, \nu)\right]\left(\zeta_{0}+\xi_{0}^{R}\right)=0, \\
\xi_{0}=\zeta_{0}+\xi_{0}^{R}, \quad \xi_{0}^{R} \in \Re\left(\Delta\left(\omega_{0}, 0,0\right)\right) .
\end{gathered}
$$

Applying the implicit function theorem to $(\alpha 3)$ implies that there exists a smooth function $\xi_{0}^{R}=\xi_{0}^{R}\left(\omega, \mu, \nu, \zeta_{0}\right)$ satisfying $(\alpha 3)$ for $(\omega, \mu, \nu)$ in a neighborhood of $\left(\omega_{0}, 0,0\right)$. Since $(\alpha 3)$ is a linear equation, $\xi_{0}^{R}\left(\omega, \mu, \nu, \zeta_{1}\right)$ can be written as

$$
\xi_{0}^{R}\left(\omega, \mu, \nu, \zeta_{0}\right)=\delta(\omega, \mu, \nu) \zeta_{0}, \quad \delta\left(\omega_{0}, 0,0\right)=0
$$

Hence $(\alpha 3)$ and $(\alpha 4)$ are equivalent to the equation

$$
\pi_{0}\left[\Delta\left(\omega_{0}, 0,0\right)-\Delta(\omega, \mu, \nu)\right](I+\delta(\omega, \mu, \nu)) \zeta_{0}=0
$$

This implies

$$
H(\omega, \mu, \nu) \equiv\left(G(\omega, \mu, \nu) \zeta_{0}, \zeta_{0}^{*}\right)=0
$$




$$
G(\omega, \mu, \nu) \equiv\left[\Delta\left(\omega_{0}, 0,0\right)-\Delta(\omega, \mu, \nu)\right](I+\delta(\omega, \mu, \nu)) .
$$

It is easily checked that

$$
\begin{aligned}
& H\left(\omega_{0}, 0,0\right)=0, \\
& \begin{aligned}
\frac{\partial H}{\partial \omega}\left(\omega_{0}, 0,0\right) & =-i\left(\left(I-\int_{-r}^{0} \theta e^{i \omega_{0} \theta}[d \eta(\theta ; 0)]\right) \zeta_{0}, \zeta_{0}^{*}\right) \\
& =-i\left(\mathbb{M}(0) \zeta_{0}, \zeta_{0}^{*}\right)=-i \quad(\text { see }(1.17)), \\
\frac{\partial H}{\partial \mu}\left(\omega_{0}, 0,0\right) & =\left(\frac{\partial L}{\partial \mu}(0)\left(e^{i \omega_{0} *} \zeta_{0}\right), \zeta_{0}^{*}\right) \\
& =\left(F_{\mu u}(0) \zeta_{1}, \zeta_{0}^{*}\right)=\frac{d \lambda}{d \mu}(0) \quad(\text { see }(3.4)) .
\end{aligned}
\end{aligned}
$$

Define a vector function $\tilde{H}(\omega, \mu, \nu){ }^{t}(\mathbb{R e} H(\omega, \mu, \nu), \operatorname{Im} H(\omega, \mu, \nu))$. Then

$$
\begin{aligned}
& \tilde{H}\left(\omega_{0}, 0,0\right)=0, \\
& \left(\frac{\partial \tilde{H}}{\partial \omega}\left(\omega_{0}, 0,0\right), \frac{\partial \tilde{H}}{\partial \mu}\left(\omega_{0}, 0,0\right)\right)=\left(\begin{array}{cc}
0 & -1 \\
\operatorname{Re} \frac{d \lambda}{d \mu}(0) & \operatorname{Im} \frac{d \lambda}{d \mu}(0)
\end{array}\right) .
\end{aligned}
$$

Thus, by (A1) and the implicit function theorem we get smooth functions $\omega=\alpha_{0}(\nu)$, $\mu=\mu_{0}(\nu)$ and $\xi_{0}=\xi_{0}(\nu)=\zeta_{0}+\delta\left(\alpha_{0}(\nu), \mu_{0}(\nu), \nu\right) \zeta_{0}$ which satisfy $(\alpha 1)$ for $\nu$ small.

Next for the functions $\alpha_{0}(\nu)$ and $\mu_{0}(\nu)$ obtained above we shall calculate the derivatives, $\frac{d \alpha_{0}}{d \nu}(0)$ and $\frac{d \mu_{0}}{d \nu}(0)$. Differentiating the relation

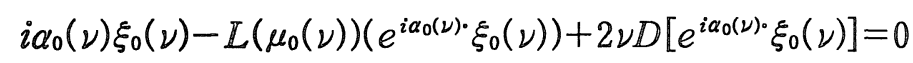

with respect to $\nu$ and putting $\nu=0$ yield

$$
\begin{aligned}
& i \frac{d \alpha_{0}}{d \nu}(0)\left\{\zeta_{0}-L(0)\left(\cdot e^{i \omega_{0} \cdot} \zeta_{0}\right)\right\}+2 D\left[e^{i \omega_{0}^{*}} \zeta_{0}\right] \\
& \quad-\frac{d \mu_{0}}{d \nu}(0) \frac{\partial L}{\partial \mu}(0)\left(e^{i \omega_{0} \cdot} \zeta_{0}\right)+\left(i \omega_{0}-L(0)\left(e^{i \omega_{0}^{*}}\right)\right) \frac{d \xi_{0}}{d \nu}(0)=0 .
\end{aligned}
$$

By putting $\nu=0$ in (1.14), (1.15), (1.17) and (3.4), we see from the above that

$$
i \frac{d \alpha_{0}}{d \nu}(0)+2\left(D \zeta_{1}, \zeta_{0}^{*}\right)-\frac{d \mu_{0}}{d \nu}(0) \frac{d \lambda}{d \mu}(0)=0
$$

holds. Hence we get 


$$
\frac{d \mu_{0}}{d \nu}(0)=\frac{2 \operatorname{Re}\left(D \zeta_{1}, \zeta_{0}^{*}\right)}{\operatorname{Re} \frac{d \lambda}{d \mu}(0)}
$$

$$
\frac{d \alpha_{0}}{d \nu}(0)=\operatorname{Im} \frac{d \lambda}{d \mu}(0) \frac{d \mu_{0}}{d \nu}(0)-2 \operatorname{Im}\left(D \zeta_{1}, \zeta_{0}^{*}\right)
$$

\section{Appendix B.}

We shall solve the equation (4.17). We assume that $\gamma_{2}$ is a root of (4.20); a similar proof will hold if $\gamma_{2}$ is a root of (4.21). In this case, $\left(c_{1}, c_{1}^{\prime}\right) \neq 0, c_{2}=c_{2}^{\prime}=$ 0 , and the equation (4.17) can be written as

$$
\left\{\begin{array}{l}
W_{1}^{(1)}=\left(-\gamma_{2}+\nu_{2}\left(D \zeta_{1}, \zeta_{0}^{*}\right)+B_{1}\right) \eta_{1}+B_{1} \eta_{1}^{\prime}-\hat{\gamma}\left(c_{1}+\eta_{1}\right)+O(\varepsilon), \\
W_{1}^{(2)}=\left(-\gamma_{2}+\nu_{2}\left(D \bar{\zeta}_{1}, \overline{\zeta_{0}^{*}}\right)+\bar{B}_{1}\right) \eta_{1}^{\prime}+\bar{B}_{1} \eta_{1}-\hat{\gamma}\left(c_{1}^{\prime}+\eta_{1}^{\prime}\right)+O(\varepsilon), \\
W_{2}^{(1)}=\left(-\gamma_{2}+B_{1}\right) \eta_{2}+B_{1} \eta_{2}^{\prime}-\hat{\gamma} \eta_{2}+O(\varepsilon), \\
W_{2}^{(2)}=\left(-\gamma_{2}+\bar{B}_{1}\right) \eta_{2}^{\prime}+\bar{B}_{1} \eta_{2}-\bar{\gamma} \eta_{2}^{\prime}+O(\varepsilon) .
\end{array}\right.
$$

First assume that $\gamma_{2}$ is a complex root of (4.20), and consider the case $c_{1} \neq 0$. Then we put $\eta_{1}=0$. Let $W \equiv\left(W_{1}^{(1)}, W_{1}^{(2)}, W_{2}^{(1)}, W_{2}^{(2)}\right)$ and write $\delta \equiv\left(D \zeta_{1}, \zeta_{0}^{*}\right)$, $\delta_{1} \equiv \operatorname{Re} \delta, \delta_{2} \equiv \operatorname{Im} \delta, b_{1} \equiv \operatorname{Re} B_{1}, b_{2} \equiv \operatorname{Im} B_{1}$. From $(\beta 1)$,

$$
\begin{aligned}
\operatorname{det}( & \left.\frac{\partial W}{\partial\left(\bar{\gamma}, \eta_{1}^{\prime}, \eta_{2}, \eta_{2}^{\prime}\right)}\right)_{\mid}\left(\varepsilon, \hat{\gamma}, \eta_{1}^{\prime}, \eta_{2}, \eta_{2}^{\prime}\right)=(0,0,0,0,0) \\
\quad & =-\left\{c_{1}\left(-\gamma_{2}+\nu_{2} \bar{\delta}+\bar{B}_{1}\right)-c_{1}^{\prime} B_{1}\right\}\left(\gamma_{2}^{2}-2 b_{1} \gamma_{2}\right) \\
& =-2 c_{1} \gamma_{2}\left\{-\gamma_{2}+\nu_{2}\left(\delta_{1}+b_{1}\right)\right\}\left(\gamma_{2}-2 b_{1}\right) \quad \text { (from (4.18a)). }
\end{aligned}
$$

Thus, if $\gamma_{2} \neq \nu_{2}\left(\delta_{1}+b_{1}\right)$ (which means that $\gamma_{2}$ is not a double root of (4.20)) and $\gamma_{2}$ $\neq 0,2 b_{1}\left(=\beta_{2}\right)$, then $(\beta 2)$ is not zero. This implies that the implicit function theorem applies to $(\beta 1)$, to obtain a solution $\left(\hat{\gamma}, 0, \eta_{1}^{\prime}, \eta_{2}, \eta_{2}^{\prime}\right)=\left(\hat{\gamma}(\varepsilon), 0, \eta_{1}^{\prime}(\varepsilon), \eta_{2}(\varepsilon), \eta_{2}^{\prime}(\varepsilon)\right)$ of $(\beta 1)$ for sufficiently small $\varepsilon$.

If $c_{1}=0\left(c_{1}^{\prime} \neq 0\right)$, then putting $\eta_{1}^{\prime}=0$ and replacing $\eta_{1}^{\prime}$ by $\eta_{1}$ in the above proof yield a similar conclusion.

Finally let $\gamma_{2}$ be a real root of (4.20). Then we seek a real valued solution of (4.3), which implies $c_{i}^{\prime}=\bar{c}_{i} \eta_{i}^{\prime}=\bar{\eta}_{i}(i=1,2)$. Using a similar argument found in $[19$; Appendix], one can solve the equation $(\beta 1)$; the details of the proof will be left to the readers. 


\section{Referemces}

[1] M. Ashkenazi and H. G. Othmer, Spatial Patterns in Coupled Biochemical Oscillators, $J$. Math. Biol. 5 (1978), 305-350.

[2] S. Chow, Existence of Periodic Solutions of Autonomous Functional Differential Equations, J. Differential Equations 15 (1974), 350-378.

[3] S. Chow and J. Mallet-Paret, Integral Averaging and Bifurcation, J. Differential Equations 26 (1977), 112-159.

[4] J. M. Cushing, Integrodifferential Equations and Delay Models in Population Dynamics, Lecture Notes in Biomathematics 20, Springer Verlag, New York 1977.

[5] H. A. Dekline, E. Kennedy and N. MacDonald, A Study of Coupled Systems of Nonlinear Oscillators, preprint.

[6] T. Erneux and M. Herschkowitz-Kaufman, Bifurcation Diagram of a Mcdel Chemical Reaction-I : Stability Changes of Time-Periodic Solution, Bull. Math. Biol. 41(1979), 2138.

[7] J. Hale, Theory of Functional Differential Equations,Springer Verlag, New York 1977.

[8] B. P. Hassard, N. D. Kazarinoff and Y. H. Wan, Theory and Applications of Hopf Bifurcation, London Math. Sci., Lecture Note \&1 (1981).

[9] G. E. Hutchinson, Circular Causal Systems in Ecology, Ann. N. Y. Acad. Sci. 50 (1948), 221-246.

[10] G. Iooss and D. D. Joseph, Elementary Stability and Bifurcation Theory, Springer Verlag, New York 1980.

[11] G. S. Jones, The Existence of Periodic Solution of $\mathrm{f}^{\prime}(x)=-\alpha \mathrm{f}(x-1)\{1+\mathrm{f}(x)\}$, J. Math. Anal. Appl. 5 (1962), 435-450.

[12] J. Kaplan and J. A. Yorke, Ordinary Differential Equations which yield Periodic Solutions of Differential Delay Equations, J. Math. Anal. Appl. 48 (1974), 317-324.

[13] On the Stability of a Periodic Solution of a Differential Delay Equation, SIAM J. Math. Anal. 6 (1975), 268-282.

[14] M. Kawato and R. Suzuki, Synergism and Antagonism of Neurons Caused by an Electrical Synapse, Biol. Cybernetics 34 (1979), 81-89.

[15] - Two Coupled Newral Oscillators as a Model of the Circadian Pacemaker, $J$. Theoret. Biol. $\$ 6$ (1980), 547-575.

[16] J. Lin and P. B. Kahn, Phase and Amplitude Instability in Delay-Diffusion Population Models, J. Math. Biol. $\mathbb{1 3}$ (1982), 383-393.

[17] N. MacDonald, Time Lags in Biological Models, Lecture Notes in Biomathematics 27, Springer Verlag, New York 1978.

[18] J. E. Marsden and M. McCracken, The Hopf Bifurcation and its Applications, Springer Verlag, Applied Mathematical Sciences $\mathbb{1 9}$, New York 1976; S. Smale, A Mathematical Model of Two Cells via Turing's Equation, 354-367.

[19] Y. Morita, Destabilization of Periodic Solutions Arising in Delay-Diffusion Systems in Several Space Dimensions, to appear in Japan J. of Appl. Math.

[20] R. Nussbaum, Periodic Solutions of Some Nonlinear Autonomous Functional Differential 
Equations II, J. Differential Equations 14 (1973), 360-394.

[21] O. E. Rössler, Chemical Turbulence: Chaos in a Simple Reaction-Diffusion System, $Z$. Naturforsch, 31a (1976), 1168-1172.

[22] I. Schreiber and M. Marek, Strange Attractors in Coupled Reaction-Diffusion Cells, Phisica 5D (1982), 258-272.

[23] V. Torre, Synchronization of Non-Linear Biochemical Oscillators Coupled by Diffusion, Biol. Cybernetics 17 (1975), 137-144.

[24] J. J. Tyson, Some Further Studies of Nonlinear Oscillations in Chemical Systems, J. Chem. Phys. 58 (1973); 3919-3930.

[25] E. M. Wright, A Nonlinear Differential Difference Equation, J. Reine Angew Math. 194 (1955), 66-87. 